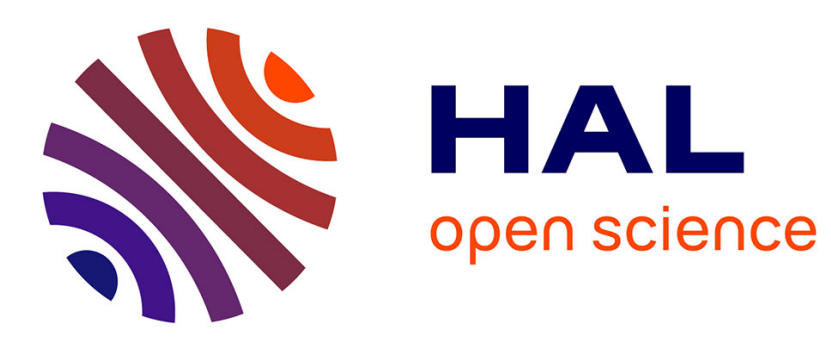

\title{
Impact of demography on extinction/fixation events
}

\author{
Camille Coron, Sylvie Méléard, Denis Villemonais
}

\section{To cite this version:}

Camille Coron, Sylvie Méléard, Denis Villemonais. Impact of demography on extinction/fixation events. Journal of Mathematical Biology, 2019, 78 (3), pp.549-577. 10.1007/s00285-018-1283-1 . hal-01514977v2

\section{HAL Id: hal-01514977 \\ https://hal.science/hal-01514977v2}

Submitted on 4 Jan 2019

HAL is a multi-disciplinary open access archive for the deposit and dissemination of scientific research documents, whether they are published or not. The documents may come from teaching and research institutions in France or abroad, or from public or private research centers.
L'archive ouverte pluridisciplinaire HAL, est destinée au dépôt et à la diffusion de documents scientifiques de niveau recherche, publiés ou non, émanant des établissements d'enseignement et de recherche français ou étrangers, des laboratoires publics ou privés. 


\title{
Impact of demography on extinction/fixation events
}

\author{
C. Coron* S. Méléard† D. Villemonais ${ }^{\ddagger}$
}

July 2, 2018

\begin{abstract}
In this article we consider diffusion processes modeling the dynamics of multiple allelic proportions (with fixed and varying population size). We are interested in the way alleles extinctions and fixations occur. We first prove that for the Wright-Fisher diffusion process with selection, alleles get extinct successively (and not simultaneously), until the fixation of one last allele. Then we introduce a very general model with selection, competition and Mendelian reproduction, derived from the rescaling of a discrete individual-based dynamics. This multi-dimensional diffusion process describes the dynamics of the population size as well as the proportion of each type in the population. We prove first that alleles extinctions occur successively and second that depending on population size dynamics near extinction, fixation can occur either before extinction almost surely, or not. The proofs of these different results rely on stochastic time changes, integrability of one-dimensional diffusion processes paths and multi-dimensional Girsanov's tranform.
\end{abstract}

Keywords: population dynamics and population genetics, demography and extinction, allelic fixation, diffusion processes, path integrability, diffusion absorption

\section{Introduction - A demo-genetic model}

This paper is motivated by concerns in conservation biology and more specifically by assessing conditions for the maintenance of biodiversity in populations facing extinction. Classical population genetics models like the Wright-Fisher model, the Moran model or the Wright-Fisher diffusion for instance assume a constant population size, which is then introduced as a key parameter of these models. In contrast, when conservation biology issues, one needs to understand the behaviour of populations facing extinction or composed with only a few individuals. Notably, specific phenomena such as inbreeding ([4]) and mutational meltdown $([20])$, or changes in interactions between individuals $([26])$ are observed in small populations. To study these kinds of phenomena one therefore needs to consider models that allow to take into account and study the joint dynamics of both the demography and the genetic composition of a population. Our aim in this paper is more specifically to understand the impact of demography, and in particular of extinction, on allele extinction (or fixation).

We first study the dynamics of the progressive loss of genetic diversity in a classical population genetics context (constant population size) and second the impact of the fluctuations of population demography on genetic diversity. We consider a population composed of hermaphroditic diploid individuals characterized by their genotype at one locus presenting $L$ possible alleles. The dynamics is modeled by a multidimensional diffusion process. The first study (Section 2) concerns the $L$-allelic diploid Wright-Fisher diffusion (see [12], Chap. 10). We prove (Theorem 2.1) that in this model the alleles disappear successively until the fixation of a single last allele. Therefore fixed population size induces a progressive loss of genetic diversity. The proof is done by induction on $L$ and is based on successive time changes and a criterion for perpetual integrals finiteness.

\footnotetext{
* Laboratoire de Mathématiques d'Orsay, Univ. Paris-Sud, CNRS, Université Paris-Saclay, 91405 Orsay, France

${ }^{\dagger}$ CMAP, Ecole Polytechnique, CNRS, route de Saclay, 91128 Palaiseau, France

¥IECL, UMR 7502, CNRS, Université de Lorraine, Vandœuvre-lès-Nancy et Inria, TOSCA team, Villers-lès-Nancy, France
} 
The rest of the article focuses on the impact of demography on genetic diversity. We introduce a diffusion process $\left(N(t), X^{2}(t), X^{3}(t), \ldots, X^{L}(t)\right)_{t \geq 0}$ giving the joint behavior of the population size and the proportions of types $2,3, \ldots, L$. Note that $X^{1}=1-\sum_{i=2}^{L} X^{i}$ is the proportion af allele 1 . This diffusion is derived from a slow-fast rescaling of a diploid multi-type birth and death process (see Appendix A). This individual-based model includes Mendelian reproduction, competition, and selection on birth, natural death and competition parameters. Since individuals are diploid, their genotypes are of the form $i j$ where $i, j \in\{1, \ldots, L\}$.

The infinitesimal generator of the considered diffusion process is given for $\left.\left.\left(n, x_{2}, \ldots, x_{L}\right) \in\right] 0,+\infty\right) \times$ $\left\{\left(x_{2}, \ldots, x_{L}\right) \in[0,1] ; x_{2}+\ldots+x_{L} \leq 1\right\}$ and any function $f \in \mathcal{C}_{b}^{2}\left([0,+\infty) \times\left\{\left(x_{2}, \ldots, x_{L}\right) \in[0,1] ; x_{2}+\ldots+x_{L} \leq\right.\right.$ $1\}, \mathbb{R})$ by

$$
\begin{aligned}
& \mathcal{L}_{1} f\left(n, x_{2}, \ldots, x_{L}\right)=n\left(\rho-\alpha n+\sum_{1 \leq i, j \leq L}\left(s_{i j}-n \sum_{1 \leq k, l \leq L} c_{i j, k l} x_{k} x_{l}\right) x_{i} x_{j}\right) \frac{\partial f}{\partial n}\left(n, x_{2}, \ldots, x_{L}\right) \\
& +\gamma n \frac{\partial^{2} f}{\partial n^{2}}\left(n, x_{2}, \ldots, x_{L}\right) \\
& \quad+\sum_{i=2}^{L}\left[x_{i} \sum_{j=1}^{L} \sum_{k=1}^{L} x_{j} x_{k}\left[\left(s_{i k}-s_{j k}\right)-n \sum_{1 \leq l, m \leq L}\left(c_{i k, m l}-c_{j k, m l}\right) x_{m} x_{l}\right]\right] \frac{\partial f}{\partial x_{i}}\left(n, x_{2}, \ldots, x_{L}\right) \\
& \quad+\sum_{i=2}^{L} \gamma \frac{x_{i}\left(1-x_{i}\right)}{2 n} \frac{\partial^{2} f}{\partial x_{i}^{2}}\left(n, x_{2}, \ldots, x_{L}\right)-\sum_{i \neq j \in \llbracket 2, N \rrbracket} \gamma \frac{x_{i} x_{j}}{2 n} \frac{\partial^{2} f}{\partial x_{i} \partial x_{j}}\left(n, x_{2}, \ldots, x_{L}\right) .
\end{aligned}
$$

Here $x_{1}=1-x_{2}-\ldots-x_{L}$ is the proportion of allele $1, \rho \in \mathbb{R}$ is the natural growth rate of genotype 11 and $s_{i j}$ quantifies the selective advantage of genotype $i j$ for $i, j \in\{1, \ldots, L\}$ (the higher is $s_{i j}$, the more advantageous is genotype $i j) ; s_{11}=0$ by convention. The parameter $\alpha+c_{i j, k l}>0$ quantifies the competition pressure of genotype $k l$ on genotype $i j$ (for example due to limitation of resources) and $c_{11,11}=0$ by convention. The allelic diffusion parameter $\gamma>0$ scales the speed at which birthand-death events occur. The existence and uniqueness properties of this process are given in Appendix A. The Model (1) dramatically generalizes the classical genetic models by considering an arbitrary number of alleles under different types of selection. Let us note the interplay between allelic repartition and demography through differences in competition parameters. In the mean field case with constant competition pressure $\left(c_{i j, k l}=0\right.$ for any $\left.i, j, k, l\right)$, this model is a stochastically varying population size version of the general Wright-Fisher model introduced in [12].

If $s_{i j}=0$ for all $i, j \geq 1$, the model is neutral, since alleles are exchangeable.

If $s_{i j}=\frac{1}{2}\left(s_{i}+s_{j}\right)$ for all $i, j$, which corresponds to additive selection, the generator becomes

$$
\begin{aligned}
& \mathcal{L}_{1} f\left(n, x_{2}, \ldots, x_{L}\right)=n\left(\rho-\alpha n+\sum_{i=2}^{L} s_{i} x_{i}\right) \frac{\partial f}{\partial n}\left(n, x_{2}, \ldots, x_{L}\right)+\gamma n \frac{\partial^{2} f}{\partial n^{2}}\left(n, x_{2}, \ldots, x_{L}\right) \\
& \quad+\sum_{i=2}^{L} x_{i}\left(s_{i}-\sum_{j=1}^{L} x_{j} s_{j}\right) \frac{\partial f}{\partial x_{i}}\left(n, x_{2}, \ldots, x_{L}\right) \\
& \quad+\sum_{i=2}^{L} \gamma \frac{x_{i}\left(1-x_{i}\right)}{2 n} \frac{\partial^{2} f}{\partial x_{i}^{2}}\left(n, x_{2}, \ldots, x_{L}\right)-\sum_{i \neq j \in \llbracket 2, N \rrbracket} \gamma \frac{x_{i} x_{j}}{2 n} \frac{\partial^{2} f}{\partial x_{i} \partial x_{j}}\left(n, x_{2}, \ldots, x_{L}\right) .
\end{aligned}
$$

Let us note that this generator is close to the one we would obtain in an haploid case, except that the denominator $2 n$ in the diffusion coefficients would be $n$, which changes the dynamics.

What is more, the system (1) writes as (2) with any $s_{i}$ replaced by $S_{i}$ defined by

$$
S_{i}=\sum_{k=1}^{L} s_{i k} x_{k}-n \sum_{1 \leq k, l, m \leq L} c_{i k, m l} x_{m} x_{l} x_{k} .
$$

The coefficient $S_{i}$ is the true selective advantage of allele $i$ in our general framework. It takes into account both diploid individual (genetic) selection and environmental pressure between individuals. 
In Model (1), population size goes almost-surely to 0 in finite time. We prove (Theorem 3.1) that, almost surely, the fixation of a (non given) single allele occurs before the extinction time and after the successive extinctions of the other alleles. The proof of this result is deduced from that of Theorem 2.1 using time changes and multi-dimensional Girsanov's transform.

The diffusion processes defined in (1) comes from a specific scaling in the individual-based initial model linking the population size and the demographic parameters in an allometric scale explained by the metabolic theory which relates the individuals characteristics and their mass (Cf. [3], [24], [13]). This leads in the limit to systems in which the organisms with short lives and fast reproduction create a demographic stochasticity modeled by the diffusion (Cf Champagnat et al. [4]). In the case where some specific density-dependence impacts the birth and death rates, we can obtain a different scaling leading to different population size diffusion coefficients. In Section 4 we explore the impact of the demography on allele fixation and therefore on the maintenance of biodiversity. In particular, we exhibit examples of population size dynamics for which extinction occurs before fixation of alleles with positive probability (Theorem 4.1 and Figures 1 and 2). This result implies a maintenance of genetic diversity at all times, for the considered population, and shows the main influence of demographic stochasticity on biodiversity.

Our proofs and results repeatedly rely on the study of quantities of the form $\int_{0}^{T_{0}} f\left(Z_{s}\right) d s$ (which are referred to as perpetual integrals [24]), for a nonnegative (one-dimensional) diffusion process $Z$ and $T_{0}$ its hitting time of 0 , or $\int_{0}^{T_{0} \wedge T_{1}} f\left(X_{s}\right) d s$, for a diffusion process $X \in[0,1]$ and $T_{0}, T_{1}$ its hitting times of 0 and 1. More specifically, we need to know whether such integrals are finite or not. In Appendix B, we state and prove a general criterion involving a necessary and sufficient condition based on the scale function and speed measure of the nonnegative (one-dimensional) diffusion process $Z$, which ensures that the integral $\int_{0}^{T_{0}} f\left(Z_{s}\right) d s$ is finite almost surely or infinite almost surely.

Notation: -In the following the state space will be denoted by

$$
S=] 0,+\infty) \times\left\{\left(x_{2}, \ldots, x_{L}\right) \in[0,1] ; x_{2}+\ldots+x_{L} \leq 1\right\}
$$

and its interior will be denoted by $\stackrel{\circ}{S}$.

-We denote by $T_{z}$ the hitting time of $z \in[0,+\infty)$ by the process $Z$ :

$$
T_{z}=\inf \left\{t \geq 0, Z_{t}=z\right\} .
$$

When the process $Z$ has to be specified, this time will be denoted $T_{z}^{Z}$.

\section{Successive fixations for the multi-allelic neutral Wright-Fisher diffusion}

In this section we consider a neutral $L$-type Wright-Fisher diffusion (Ethier-Kurtz [12], pp. 435 - 439) describing the dynamics of the respective proportions of $L$ alleles in a population with fixed size. We are interested in the study of alleles extinctions in this model.

Let us define by $X_{t}^{i}$ the proportion of allele $i$ in the population at time $t$. Since by definition $X_{t}^{1}+$ $\cdots+X_{t}^{L}=1$ for any time $t$, it is enough to study the dynamics of the process $\left(X_{t}^{1}, \cdots, X_{t}^{L-1}\right)_{t \geq 0}$. The Wright-Fisher diffusion (see for example [12, Chap. 10]) is a stochastic diffusion whose infinitesimal generator $\mathcal{L}_{1}$ is defined for all $\left(x_{1}, \ldots x_{L-1}\right) \in\left\{\left(x_{1}, \ldots, x_{L-1}\right) \in[0,1]^{L-1} ; x_{1}+\ldots+x_{L-1} \leq 1\right\}$ and for all function $f \in \mathcal{C}^{2}\left(\left\{\left(x_{1}, \ldots, x_{L-1}\right) \in[0,1]^{L-1} ; x_{1}+\ldots+x_{L-1} \leq 1\right\}, \mathbb{R}\right)$ by

$$
\mathcal{L}_{1} f\left(x_{1}, \cdots, x_{L-1}\right)=\sum_{i=1}^{L-1} x_{i}\left(1-x_{i}\right) \frac{\partial^{2} f}{\partial x_{i}^{2}}\left(x_{1}, \cdots, x_{L-1}\right)-\sum_{i \neq j \in \llbracket 1, L-1 \rrbracket} x_{i} x_{j} \frac{\partial^{2} f}{\partial x_{i} \partial x_{j}}\left(x_{1}, \cdots, x_{L-1}\right) .
$$

Our aim is to prove the following theorem: 
Theorem 2.1. (i) One of the $L$ alleles is fixed almost surely in finite time, i.e. the random variable $\max _{i \in\{1, \cdots, L\}} X^{i}$ is absorbed at 1 in finite time almost surely.

(ii) Till that time, the population experiences successive (and non simultaneous) allele extinctions.

The proof of this theorem is based on an induction argument and relies on two lemmas.

Lemma 2.2. Let $Y$ be the process solution of

$$
d Y_{t}=\sqrt{Y_{t}\left(1-Y_{t}\right)} d B_{t} ; Y_{0} \in(0,1)
$$

where $\left(B_{t}, t \geq 0\right)$ is a standard Brownian motion. Then, setting $T_{1}=\inf \left\{t \geq 0, Y_{t}=1\right\}$, we have for any $y \in(0,1)$

$$
\mathbb{P}_{y}\left(\int_{0}^{T_{1}} \frac{1}{1-Y_{s}} d s=+\infty\right)=1 .
$$

Proof. It is well known that $Y$ reaches 0 or 1 in finite time a.s.. The process is on natural scale and the speed measure on $(0,1)$ is given by $m(d y)=\frac{2 d y}{y(1-y)}$. Setting $f(y)=1 /(1-y)$, we have $\int^{1-}(s(1)-$ $s(y)) f(y) m(d y)=+\infty$ and Theorem B.4 of Appendix B yields

$$
\mathbb{P}_{y}\left(\left\{\int_{0}^{T_{1}} \frac{1}{1-Y_{s}} d s=+\infty\right\} \cap\left\{T_{1}<T_{0}\right\}\right)=\mathbb{P}_{y}\left(T_{1}<T_{0}\right) .
$$

Since $\left\{T_{1}=+\infty\right\}=\left\{T_{0}<T_{1}\right\}$ and $1 /\left(1-Y_{t}\right)=1$ for all $t \geq T_{0}$, we get the result.

Lemma 2.3. Let $\left(X^{1}(t), \ldots, X^{L-1}(t)\right)_{t \geq 0}$ be a $L$ - 1-dimensional Wright-Fisher diffusion process, let $1-X^{L}(t)=X^{1}(t)+\ldots+X^{L-1}(t)$ for all time $t \geq 0$, and define the time change $\tau$ on $[0,+\infty)$ such that $\int_{0}^{\tau(t)} \frac{1}{1-X_{L}(s)} d s=t$ for all $t \geq 0$ (see Lemma 2.2). Now let

$$
\left(Y_{t}^{1}, Y_{t}^{2}, \ldots, Y_{t}^{L-2}\right)_{t \geq 0}=\left(\frac{X^{1}}{1-X^{L}}(\tau(t)), \ldots, \frac{X^{L-2}}{1-X^{L}}(\tau(t))\right)_{t \geq 0} .
$$

The stochastic process $\left(Y_{t}^{1}, Y_{t}^{2}, \ldots, Y_{t}^{L-2}\right)_{t \geq 0}$ is a $L$-2-dimensional Wright-Fisher diffusion process.

Proof of Lemma 2.3. Let us denote by $\tilde{\mathcal{L}}$ the infinitesimal generator of the $L$ - 1-dimensional diffusion process $\left(\frac{X^{1}}{1-X^{L}}(t), \frac{X^{2}}{1-X^{L}}(t), \ldots, \frac{X^{L-2}}{1-X^{L}}(t), 1-X^{L}(t)\right)_{t \geq 0}$. For any real-valued twice differentiable function $f$ defined on $\left\{\left(\tilde{x}_{1}, \ldots, \tilde{x}_{L-2}, 1-x_{L}\right) \in[0,1]^{L-1} ; \tilde{x}_{1}+\ldots+\tilde{x}_{L-2} \leq 1\right\}$, we may write for $x_{L} \neq 1$,

$$
\tilde{\mathcal{L}} f\left(\tilde{x}_{1}, \ldots, \tilde{x}_{L-2}, 1-x_{L}\right)=\mathcal{L}_{1}(f \circ g)\left(x_{1}, \ldots, x_{L-1}\right),
$$

where $\left(\tilde{x}_{1}, \ldots, \tilde{x}_{L-2}, 1-x_{L}\right)=g\left(x_{1}, \ldots, x_{L-1}\right)$ and, for any $\left(x_{1}, \ldots x_{L-1}\right) \in[0,1]^{L-1}$ such that $0<$ $x_{1}+\ldots+x_{L-1} \leq 1$

$$
g\left(x_{1}, \ldots, x_{L-1}\right)=\left(\frac{x_{1}}{x_{1}+\ldots+x_{L-1}}, \ldots, \frac{x_{L-2}}{x_{1}+\ldots+x_{L-1}}, x_{1}+\ldots+x_{L-1}\right) .
$$

Therefore, we obtain from Equation (3) that for $x_{L} \neq 1$,

$$
\begin{aligned}
\tilde{\mathcal{L}} f\left(\tilde{x}_{1}, \tilde{x}_{2}, \ldots, \tilde{x}_{L-2}, 1-x_{L}\right) & =\sum_{j=1}^{L-2} \frac{\gamma \tilde{x}_{j}\left(1-\tilde{x}_{j}\right)}{1-x_{L}} \frac{\partial^{2} f}{\partial \tilde{x}_{j}^{2}}\left(\tilde{x}_{1}, \tilde{x}_{2}, \ldots, \tilde{x}_{L-2}, 1-x_{L}\right) \\
& -\sum_{j \neq k \in \llbracket 1, L-2 \rrbracket} \frac{\gamma \tilde{x}_{j} \tilde{x}_{k}}{1-x_{L}} \frac{\partial^{2} f}{\partial \tilde{x}_{j} \partial \tilde{x}_{k}}\left(\tilde{x}_{1}, \tilde{x}_{2}, \ldots, \tilde{x}_{L-2}, 1-x_{L}\right) \\
& +\gamma x_{L}\left(1-x_{L}\right) \frac{\partial^{2} f}{\partial\left(1-x_{L}\right)^{2}}\left(\tilde{x}_{1}, \tilde{x}_{2}, \ldots, \tilde{x}_{L-2}, 1-x_{L}\right)
\end{aligned}
$$

which gives the result since $d \tau(t)=\left(1-X^{L}(t)\right) d t$. 
Proof of Theorem 2.1. We prove both results by induction on $L$. (i) is a well known result in the case $L=2$. Now for $L$ alleles, note that the proportion of allele 1 follows a 1-dimensional Wright-Fisher diffusion. Therefore allele 1 gets fixed or disappears almost surely in finite time. If allele 1 gets fixed then one of the $L$ alleles gets fixed. If allele 1 gets lost then from its (almost surely finite) extinction time, the population follows a $L-1$-type Wright-Fisher diffusion, therefore one of the $L-1$ remaining alleles gets fixed almost surely in finite time, using the induction assumption.

We now prove $(i i)$ (which is trivial when $L=2$ ). We have $\int_{0}^{T_{1}^{L}} \frac{1}{1-X_{s}^{L}} d s=+\infty$ from Lemma 2.2. Let us consider the time change $\tau(t)$, defined for all $t \in[0,+\infty)$ by $\int_{0}^{\tau(t)} \frac{1}{1-X_{s}^{L}} d s=t$. Note that for $t \in[0,+\infty)$, $X_{L}(\tau(t))<1$.

Therefore we can define the stochastic process $Y_{t}=\left(Y_{t}^{1}, \ldots, Y_{t}^{L-2}\right)_{t \geq 0}$ such that $Y_{t}^{i}=\frac{X^{i}}{1-X^{L}}(\tau(t))$ for all $1 \leq i \leq L-2$ and for any $t \in[0,+\infty)$. From Lemma 2.3 , the stochastic process $\left(Y_{t}^{1}, Y_{t}^{2}, \ldots, Y_{t}^{L-2}\right)_{t \geq 0}$ is a $L-2$ dimensional Wright-Fisher diffusion process. By induction assumption, this diffusion process experiences $L-2$ successive and non simultaneous extinctions, at times denoted by $S_{1}^{Y}<\ldots<S_{L-2}^{Y}<$ $+\infty$. Therefore $\tau\left(S_{1}^{Y}\right)<\ldots<\tau\left(S_{L-2}^{Y}\right)<\tau(+\infty)=T_{1}^{L}$. Under the event $\left\{T_{1}^{L}<+\infty\right\}$, the times $\tau\left(S_{1}^{Y}\right), \ldots, \tau\left(S_{L-2}^{Y}\right)$ and $T_{1}^{L}$ correspond to the $L-1$ extinction times experienced by the population, which gives the result, since $\mathbb{P}\left(\cup_{i=1}^{L}\left\{T_{1}^{i}<+\infty\right\}\right)=1$ from $(i)$.

\section{Long time behavior of the diffusion process (1)}

In this section, we focus on the stochastic diffusion process $\left(N(t), X^{2}(t), X^{3}(t), \ldots, X^{L}(t)\right)_{t \geq 0}$ whose infinitesimal generator is given in (1) and whose existence is obtained by the scaling limit of a multi-type birth-and-death process (see Appendix A, Theorem A.4 for existence and uniqueness). Here the genetic dynamics of the population depends on both the selection and the competition between individuals, and the population size dynamics depends on the allelic repartition. The following theorem generalizes the results obtained in Theorem 2.1, to this very general class of demogenetics models. The main intuition (for the proof) is that the speed of allelic extinctions is inversely proportional to population size. So we introduce an appropriate time change to compensate the population size variability.

Theorem 3.1. $\quad(i)$ The population size process $(N(t))_{t \geq 0}$ is absorbed at 0 (extinction of the population) almost surely in finite time.

(ii) One of the allele will eventually get fixed before the extinction of the population, almost surely.

(iii) Till that time, the population experiences successive (and not simultaneous) allele extinctions.

Proof. (i) From (1), using that $x_{i} \in[0,1]$ for all $i$, and setting $\bar{\rho}=\sup _{i, j}\left\{\rho+s_{i j}\right\}$ and $\underline{\alpha}=\inf _{i, j, k, l}\{\alpha+$ $\left.c_{i j, k l}\right\}$, one can easily see that the process $(N(t))_{t \geq 0}$ is stochastically dominated by the logistic Feller diffusion process $(\bar{N}(t))_{t \geq 0}$ satisfying $d \bar{N}_{t}=\bar{N}_{t}\left(\bar{\rho}-\underline{\alpha} \bar{N}_{t}\right) d t+\sqrt{2 \gamma \bar{N}_{t}} d B_{t}$ which is known to reach 0 almost surely in finite time ([16], Chapter VI.3).

(ii) and (iii). We first use a multi-dimensional Girsanov transform to reduce the study to the neutral diffusion process (for which $s_{i j}=c_{i j, k l}=0$ for all $i, j, k, l$ ). We introduce an appropriate time change to compensate the population size variability. That allows us to deduce the long time behavior of the diffusion process (1) from that of the classical Wright-Fisher diffusion process, obtained in Theorem 2.1.

The infinitesimal generator (1) writes 


$$
\begin{aligned}
\mathcal{L}_{1} f\left(n, x_{2}, \ldots, x_{L}\right) & =n\left(\rho-\alpha n+\sum_{1 \leq i, j \leq L}\left(s_{i j}-n \sum_{1 \leq k, l \leq L} c_{i j, k l} x_{k} x_{l}\right) x_{i} x_{j}\right) \frac{\partial f}{\partial n}\left(n, x_{2}, \ldots, x_{L}\right) \\
& +\gamma n \frac{\partial^{2} f}{\partial n^{2}}\left(n, x_{2}, \ldots, x_{L}\right) \\
& +\sum_{i=2}^{L} b_{i}\left(n, x_{2}, \ldots, x_{L}\right) \frac{\partial f}{\partial x_{i}}\left(n, x_{2}, \ldots, x_{L}\right) \\
& +\frac{1}{2} \sum_{i, j \in \llbracket 2, N \rrbracket} a\left(n, x_{2}, \ldots, x_{L}\right)_{i j} \frac{\partial^{2} f}{\partial x_{i} \partial x_{j}}\left(n, x_{2}, \ldots, x_{L}\right),
\end{aligned}
$$

where the diffusion matrix $a\left(n, x_{2}, x_{3}, \ldots, x_{L}\right)$ satisfies for $i \neq j$

$$
a\left(n, x_{2}, x_{3}, \ldots, x_{L}\right)_{i i}=\gamma \frac{x_{i}\left(1-x_{i}\right)}{n} \quad \text { and } \quad a\left(n, x_{2}, x_{3}, \ldots, x_{L}\right)_{i j}=-\gamma \frac{x_{i} x_{j}}{n} .
$$

Remark that this matrix is related to the covariance matrix of a $L$-1-dimensional multinomial $\left(n, x_{2}, x_{3}, \ldots, x_{L}\right)$ vector $Y: a\left(n, x_{1}, \ldots, x_{L}\right)=\gamma \operatorname{Cov}\left(\left(Y_{2}, \ldots, Y_{L}\right) / n\right)$. Therefore it is a symmetric positive semi-definite matrix. The vector $b$ is defined by

$$
b_{i}\left(n, x_{2}, \ldots, x_{L}\right)=x_{i} \sum_{j=1}^{L} \sum_{k=1}^{L} x_{j} x_{k}\left[\left(s_{i k}-s_{j k}\right)-n \sum_{1 \leq l, m \leq L}\left(c_{i k, m l}-c_{j k, m l}\right) x_{m} x_{l}\right] .
$$

We first prove that for all $\left(n, x_{2}, \ldots, x_{L}\right) \in \stackrel{\circ}{S}, a\left(n, x_{2}, \ldots, x_{L}\right)$ is an invertible matrix.

Lemma 3.2. Assume that $n \neq 0$, then

$$
\operatorname{det}(a)=\frac{1}{n^{L-1}}\left(1-\sum_{i=2}^{L} x_{i}\right) \prod_{i=2}^{L} x_{i}
$$

Proof. It is well known that $\operatorname{det}(a)$ is a polynomial of degree less than $2 L-2$. It is obvious that any $x_{i}$, $i=2, \ldots, L$, is a factor of $\operatorname{det}(a)$. Moreover adding all columns, we also obtain that $\left(1-\sum_{i=2}^{L} x_{i}\right)=x_{1}$ factorizes $\operatorname{det}(a)$. The derivative of $\operatorname{det}(a)$ is of degree one in any variable $x_{i}$, since it is a multilinear form on its columns whose derivatives are of degree one. The conclusion follows by computing the determinant with $x_{i}=1 / L$ (which allows us to check that the value of the dominating constant is $1 / n^{L-1}$ ).

We remark that $a\left(n, x_{2}, \ldots, x_{L}\right)=\tilde{a}\left(x_{2}, \ldots, x_{L}\right) / n$ where the second derivative of $\tilde{a}$ is bounded. Then from Theorem 5.2.3 of Stroock-Varadhan [25], there exists a Lipschitz square root $\tilde{\sigma}$ of the matrix $\tilde{a}$.

Let us note that $b_{i}\left(n, x_{2}, \ldots, x_{L}\right)=x_{i}\left(S_{i}-\sum_{j=2}^{L} S_{j} x_{j}\right)$ where

$$
S_{i}\left(n, x_{2}, \ldots, x_{L}\right)=\sum_{k=1}^{L} s_{i k} x_{k}-n \sum_{k, l, m} c_{m l, i k} x_{m} x_{l} x_{k} .
$$

We have the remarkable identity: If $\Sigma$ denotes the vector of coordinates $S_{i}\left(n, x_{2}, \ldots, x_{L}\right), i=2, \ldots, L$, then

$$
a\left(n, x_{2}, \ldots, x_{L}\right) . \Sigma=\frac{\gamma}{n} b\left(n, x_{2}, \ldots, x_{L}\right) .
$$

Then for $(n, x) \in \stackrel{\circ}{S}$,

$$
\begin{aligned}
\left\|\sigma^{-1}\left(n, x_{2}, \ldots, x_{L}\right) b\left(n, x_{2}, \ldots, x_{L}\right)\right\|^{2} & =<b\left(n, x_{2}, \ldots, x_{L}\right), a^{-1}\left(n, x_{2}, \ldots, x_{L}\right) b\left(n, x_{2}, \ldots, x_{L}\right)> \\
& =\frac{n}{\gamma}<b\left(n, x_{2}, \ldots, x_{L}\right), \Sigma>.
\end{aligned}
$$


Therefore there exists a constant $C>0$ such that for all $\left(n, x_{2}, \ldots, x_{L}\right) \in S$,

$$
\left\|\sigma^{-1}\left(n, x_{2}, \ldots, x_{L}\right) b\left(n, x_{2}, \ldots, x_{L}\right)\right\|^{2} \leq C\left(1+n^{2}\right) .
$$

Let $\left(N, X^{2}, \ldots, X^{L}\right)$ be solution to the stochastic differential system

$$
\left\{\begin{array}{l}
d N_{t}=\sqrt{\gamma N_{t}} d B_{t}^{1}+N_{t}\left(\rho-\alpha N_{t}+\sum_{i=2}^{L} S_{i}\left(N_{t}, X_{t}^{2}, \ldots, X_{t}^{L}\right) X_{t}^{i}\right) d t \quad ; \quad\left(N_{0}, X_{0}\right) \in \stackrel{\circ}{S} \\
d X_{t}=\sigma\left(N_{t}, X_{t}\right) d B_{t}+b\left(N_{t}, X_{t}\right) d t
\end{array}\right.
$$

where $X=\left(X^{2}, \ldots, X^{L}\right)$ and $B^{1}$ and $B$ are two independent Brownian motions respectively one and $L-1$-dimensional. The system is well defined as soon as the solutions stay in $\stackrel{\circ}{S}$ and then for any time $t<T_{0}^{N} \wedge T_{0}^{X^{1}} \wedge T_{0}^{X^{2}} \wedge \ldots \wedge T_{0}^{X^{L}}$, where $X^{1}=1-X^{2}-\ldots-X^{L}$.

We now use the following $L$-dimensional Girsanov transformation ([16], p. 192). Let us introduce $k \in \mathbb{N}$ and define $\tau_{k}=T_{0}^{N} \wedge T_{k}^{N} \wedge T_{0}^{X^{1}} \wedge T_{0}^{X^{2}} \wedge \ldots \wedge T_{0}^{X^{L}}$. We introduce the exponential martingale $\mathcal{E}(M)_{t \wedge \tau_{k}}$ where for any $t \leq \tau_{k}$,

$$
\begin{aligned}
& M_{t}=-\left(\left(\sum_{i=2}^{L} S_{i}\left(N_{s}, X_{s}^{2}, \ldots, X_{s}^{L}\right) \int_{0}^{t} X_{s}^{i} \sqrt{\frac{N_{s}}{\gamma}}\right) d B_{s}^{1}\right. \\
&\left.+\sum_{i=2}^{L} \int_{0}^{t} \sigma^{-1}\left(N_{s}, X_{s}^{2}, \ldots, X_{s}^{L}\right) b\left(N_{s}, X_{s}^{2}, \ldots, X_{s}^{L}\right) d B_{s}\right) .
\end{aligned}
$$

For each $k$, the martingale $\mathcal{E}(M)_{t \wedge \tau_{k}}$ is uniformly integrable, thanks to (6). Under the probability $\mathbb{Q}$ such that $\left.\frac{d \mathbb{Q}}{d \mathbb{P}}\right|_{\mathcal{F}_{t}}=\mathcal{E}(M)_{t}$, the process $\left(\widetilde{B}^{1}, \widetilde{B}\right)=\left(B^{1}-\left\langle B^{1}, M\right\rangle, B-\langle B, M\rangle\right)$ is a $L$-dimensional Brownian motion, and the process $\left(N, X^{2}, \ldots, X^{L}\right)$ is solution to the stochastic differential system

$$
\left\{\begin{array}{l}
d N_{t}=\sqrt{\gamma N_{t}} d \widetilde{B}_{t}^{1}+N_{t}\left(\rho-\alpha N_{t}\right) d t \quad ; \quad\left(N_{0}, X_{0}\right) \in \stackrel{\circ}{S}, \\
d X_{t}=\sigma\left(N_{t}, X_{t}\right) d \widetilde{B}_{t}
\end{array}\right.
$$

for $t<\tau_{k}$.

The end of the proof of (ii) and (iii) consists in using a time change in order to apply Theorem 2.1 (i) and (ii). Using Example 2 in Section B, we know that

$$
\int_{0}^{T_{0}^{N}} \frac{\gamma}{2 N_{s}} d s=+\infty
$$

a.s. Hence we can define the time change $\tau(t)$ defined for all $t \in[0,+\infty)$ as the unique positive real number satisfying

$$
\int_{0}^{\tau(t)} \frac{\gamma}{2 N_{s}} d s=t
$$

In particular, $\tau$ is increasing and, under $\mathbb{Q}$, the process defined for any $t$ by $\hat{X}_{t}=X_{\tau(t)}$ is a Markov process whose generator is given in (3).

Since $\tau(\cdot)$ is increasing, we deduce that, $\mathbb{Q}$-almost surely,

$$
T_{0}^{X^{1}} \wedge T_{0}^{X^{2}} \wedge \ldots \wedge T_{0}^{X^{L}}=\tau\left(T_{0}^{\hat{X}^{1}} \wedge T_{0}^{\hat{X}^{2}} \wedge \ldots \wedge T_{0}^{\hat{X}^{L}}\right)
$$

and that, up to a $\mathbb{Q}$-negligible event,

$$
\left\{T_{0}^{X^{1}} \wedge T_{0}^{X^{2}} \wedge \ldots \wedge T_{0}^{X^{L}}<T_{0}^{N}\right\}=\left\{T_{0}^{\hat{X}^{1}} \wedge T_{0}^{\hat{X}^{2}} \wedge \ldots \wedge T_{0}^{\hat{X}^{L}}<+\infty\right\} .
$$

Using Theorem 2.1, we deduce that

$$
\mathbb{Q}\left(T_{0}^{X^{1}} \wedge T_{0}^{X^{2}} \wedge \ldots \wedge T_{0}^{X^{L}}<T_{0}^{N}\right)=1
$$


Hence, one has

$$
\begin{aligned}
\mathbb{P}\left(T_{0}^{X^{1}} \wedge T_{0}^{X^{2}} \wedge \ldots \wedge T_{0}^{X^{L}}<T_{0}^{N}\right) & =\lim _{k \rightarrow+\infty} \mathbb{P}\left(T_{0}^{X^{1}} \wedge T_{0}^{X^{2}} \wedge \ldots \wedge T_{0}^{X^{L}}<T_{k}^{N} \wedge T_{0}^{N}\right) \\
& =\lim _{k \rightarrow+\infty} \mathbb{E}^{\mathbb{Q}}\left(\mathbf{1}_{T_{0}^{X} \wedge T_{0}^{X^{2}} \wedge \ldots \wedge T_{0}^{X^{L}}<T_{k}^{N} \wedge T_{0}^{N}} \mathcal{E}(-M)_{T_{k}^{N} \wedge T_{0}^{N}}\right) \\
& \geq \lim _{k \rightarrow+\infty} \mathbb{E}^{\mathbb{Q}}\left(\mathbf{1}_{T_{0}^{N}<T_{k}^{N}} \mathcal{E}(-M)_{T_{k}^{N} \wedge T_{0}^{N}}\right) \\
& =\lim _{k \rightarrow+\infty} \mathbb{P}\left(T_{0}^{N}<T_{k}^{N}\right)=1 .
\end{aligned}
$$

Using the same induction argument as in the proof of Theorem 2.1, this concludes the proof of (ii) and (iii) and hence of Theorem 3.1.

\section{Demography and maintenance of biodiversity}

The general demogenetics model (1) was obtained from a specific scaling of the parameters in the individual-based model. Other scalings will lead to different coefficients. In particular we can generalize the linear form of the size diffusion coefficient (Feller diffusion). Our aim in this section is to emphasize the importance of the variance effects, both in the demographic and in the genetic part of the system, on the long time behavior. The main question is whether one allele gets fixed almost surely before the population goes extinct. We will see that it depends on the behavior of the diffusion coefficient near extinction in the equation satisfied by the population size. The next theorem notably highlights the major effect of the demography on the maintenance of genetic diversity by giving a necessary and sufficient criterion ensuring almost sure fixation before extinction.

For simplicity we consider in this section the bi-allelic framework.

Let us consider the process $\left(N_{t}, X_{t}\right)_{t \geq 0}$ solution to the system of stochastic differential equations

$$
\left\{\begin{array}{l}
d N_{t}=\sigma\left(N_{t}\right) d B_{t}+N_{t}\left(\rho-\alpha N_{t}\right) d t, N_{0}>0, \alpha>0, \quad t<T_{0+}^{N}, \\
d X_{t}=\sqrt{\frac{X_{t}\left(1-X_{t}\right)}{f\left(N_{t}\right)}} d W_{t}
\end{array}\right.
$$

where $B, W$ are independent one-dimensional Brownian motions, $\sigma:(0,+\infty) \rightarrow(0,+\infty)$ is locally Lipschitz and $f:(0,+\infty) \rightarrow(0,+\infty)$ is locally bounded away from 0 and where

$$
T_{0+}^{N}:=\lim _{n \rightarrow+\infty} T_{1 / n}^{N}
$$

Note that $\liminf _{x \rightarrow 0} f(x)$ can be null or not, nevertheless the former case is more interesting and biologically motivated (see [8]). Note also that the system admits a pathwise unique strong solution, as will be explained in the proof of the following theorem (if $\sigma$ is only locally Hölder continuous, an adaptation of our proof leads to the weak existence and pathwise uniqueness of a solution to this system, so that the following result remains valid).

Theorem 4.1. Fixation occurs before extinction with probability one if and only if

$$
\int_{0+} \frac{y}{\sigma^{2}(y) f(y)} d y=+\infty
$$

In particular, if $f$ is the identity function, the behavior of $\sigma(N)$ near extinction plays a main role. Whereas for the usual demographic term $\sigma(N)=\sqrt{N}$ (studied in the previous sections), fixation occurs almost surely before extinction, a small perturbation of this diffusion term, taking for example $\sigma(N)=N^{(1-\varepsilon) / 2}$, $\varepsilon>0$, leads to extinction before fixation with positive probability. An example of trajectory for which fixation does not occur before extinction is given in Figure 1, and the effect of $\varepsilon$ on the probability of extinction before fixation is numerically studied in Figure 2. 
Note that the demographic term $\sigma(N)=\sqrt{N}$ can be explained from an individual-based stochastic system in a case of large size combined with accelerated birth and death. This corresponds to population dynamics with allometric demographies whose time scale is explained by the metabolic theory which relates the individuals characteristics and their mass (Cf. [3], [27], [15]). This leads in the limit to systems in which the organisms with short lives and fast reproduction create a demographic stochasticity modeled by the Brownian part (Cf Champagnat et al. [5]). In the case where some specific density-dependence impacts the birth and death rates, we can obtain, in the limit of large population, a demographic term of the form $\sigma(N)=N^{(1-\varepsilon) / 2}, \varepsilon>0$. For the mathematical statement of such limits, we refer to BansayeMéléard [2].
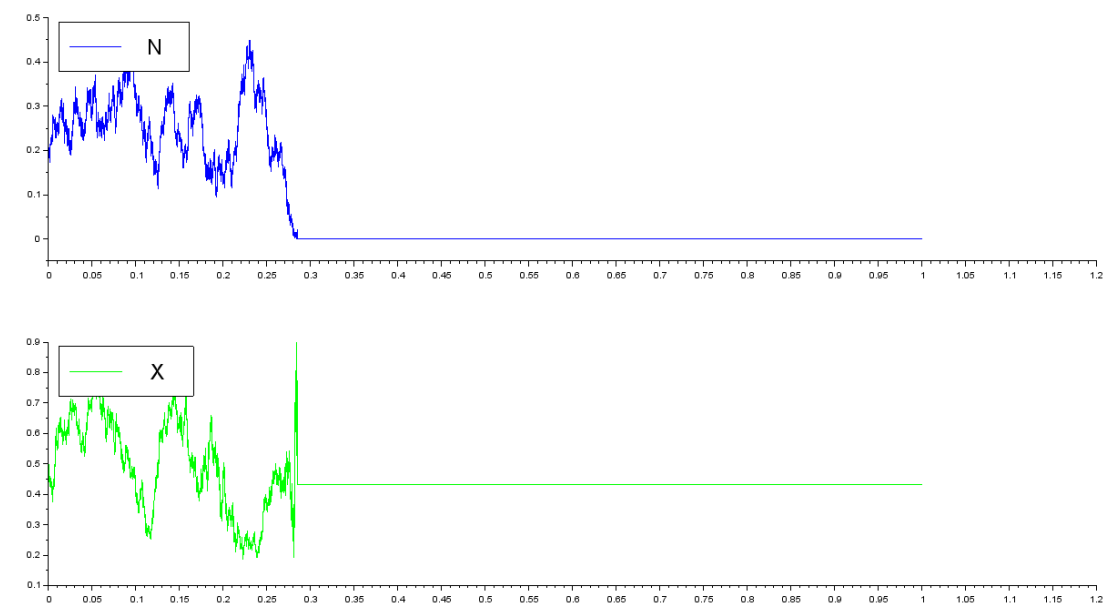

Figure 1: We plot a trajectory of the 2-dimensional diffusion process $(N, X)$ such that $d N_{t}=\sqrt{N_{t}^{(1-\varepsilon)}} d B_{t}+$ $N_{t}\left(\rho-\alpha N_{t}\right) d t$ and $d X_{t}=\sqrt{\frac{X_{t}\left(1-X_{t}\right)}{N_{t}}} d W_{t}$, with $\varepsilon=0.4, \rho=-1$ and $\alpha=0.1$. For this trajectory, fixation does not occur before extinction.

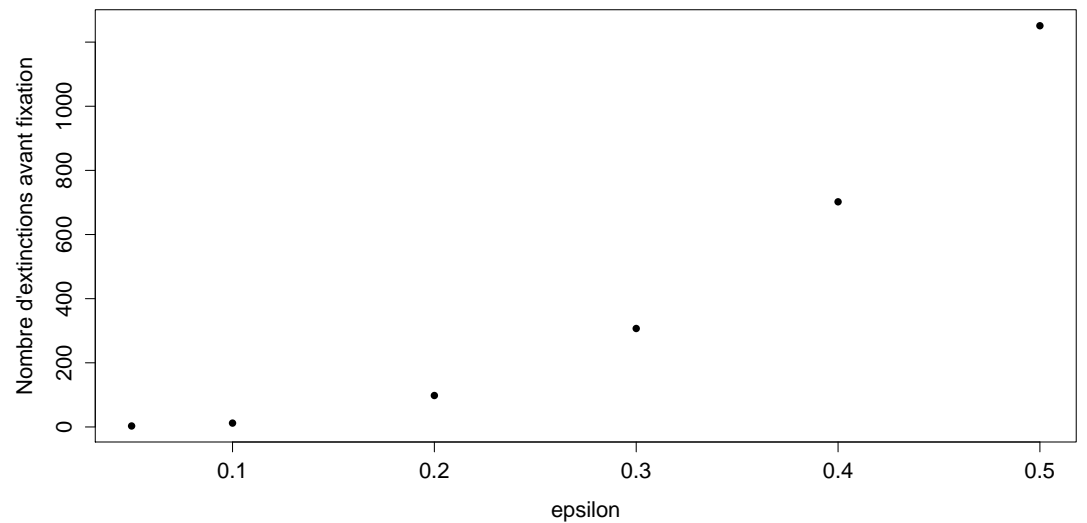

Figure 2: For different values of $\varepsilon$, we simulate 10000 trajectories of the 2-dimensional diffusion process $(N, X)$ such that $d N_{t}=\sqrt{N_{t}^{(1-\varepsilon)}} d B_{t}^{1}+N_{t}\left(r-c N_{t}\right) d t$ and $d X_{t}=\sqrt{\frac{X_{t}\left(1-X_{t}\right)}{N_{t}}}$, with $r=-1$ and $c=0.1$. We plot the number of simulations for which fixation does not occur before extinction.

Proof. Let us first prove that the system (10) admits a unique (strong) solution up to time $T_{0+}^{N}$, which in particular implies the strong Markov property used in the sequel. Given $B$ and $W$, for all $n \geq 1$, there 
exists a pathwise unique strong solution $N^{n}$ to the equation $d N_{t}^{n}=\sigma\left(N_{t}^{n}\right) d B_{t}+N_{t}^{n}\left(\rho-\alpha N_{t}^{n}\right) d t$ for all time $t<T_{1 / n}^{N}:=\inf \left\{s \geq 0, N_{s}^{n} \leq 1 / n\right\}$ (this is an immediate consequence of Theorem 3.11 p.300 in [12]). Setting $N_{t}=N_{t}^{n}$ for all $t \in\left[T_{1 / n}^{N}, T_{1 / n+1}^{N}\right.$ ), one obtains a pathwise unique strong solution to $d N_{t}=\sigma\left(N_{t}\right) d B_{t}+N_{t}\left(\rho-\alpha N_{t}\right) d t$ up to time $T_{0+}^{N}$ (in the case where $\sigma$ is only Hölder continuous, weak existence holds true, see for instance in Section 12.1 of [7]).

We define the random number

$$
T_{\max }=\int_{0}^{T_{0+}^{N}} \frac{1}{f\left(N_{s}\right)} d s
$$

and the time change $\tau(t)$, for all $t \in\left[0, T_{\max }\right)$, as the unique positive real number satisfying

$$
\int_{0}^{\tau(t)} \frac{1}{f\left(N_{s}\right)} d s=t
$$

In particular, $\tau$ is increasing and $T_{0+}^{N}=\tau\left(T_{\max }\right)$.

We define $\tilde{W}_{t}:=\int_{0}^{\tau(t)} \frac{1}{f\left(N_{s}\right)} d W_{s}$ for all $t<T_{\max }$ (which is a standard Brownian motion), and consider $\hat{X}_{t}$ the unique strong solution to

$$
d \hat{X}_{t}=\sqrt{\hat{X}_{t}\left(1-\hat{X}_{t}\right)} d \tilde{W}_{t}, \hat{X}_{0}=X_{0}, t \in\left[0, T_{\max }\right)
$$

(strong existence and pathwise uniqueness of such a solution is a consequence of Proposition 2.13 p.291 of [18]). Then the process $X_{t}:=X_{\tau^{-1}(t)}$ is a strong solution to $d X_{t}=\sqrt{\frac{X_{t}\left(1-X_{t}\right)}{f\left(N_{t}\right)}} d W_{t}$ for all $t<T_{0+}^{N}$. Pathwise uniqueness up to time $T_{1 / n, n}^{N}:=\inf \left\{t \geq 0, N_{t} \notin[1 / n, n]\right\}$ for all $n \geq 1$ is proved using the same approach as in the proof of Theorem 3.8 p.298 of [12], using the fact that $\inf _{y \in[1 / n, n]} f(y)>0$. Since $\lim _{n \rightarrow+\infty} T_{1 / n, n}^{N}=T_{0+}^{N}$ almots surely, one concludes that the system (10) admits a pathwise unique strong solution.

We denote by $\hat{T}_{F}=\inf \left\{t>0, \hat{X}_{t} \in\{0,1\}\right\}$ the (possibly infinite) absorption time of $\hat{X}$.

Assume first that $\int_{0+} \frac{y}{\sigma^{2}(y) f(y)} d y=+\infty$. In this case, using (21), we note that $s(y) \sim_{y \rightarrow 0} y s^{\prime}(y)$. Hence $T_{\max }=+\infty$ by Corollary B.3, and $\hat{X}$ reaches 0 or 1 in finite time almost surely. Then, $T_{F}=\tau\left(\hat{T}_{F}\right)<$ $\tau\left(T_{\max }\right)=T_{0+}^{N}$ (i.e. fixation occurs before extinction) almost surely.

Assume now that $\int_{0+} \frac{y}{\sigma^{2}(y) f(y)} d y<+\infty$. In this case $T_{\max }<+\infty$ with probability one by Corollary B.3. Let $\tilde{W}^{\prime}$ be a Brownian motion independent from $B$ and consider $\hat{X}^{\prime}$ the solution to the SDE $d \hat{X}_{t}^{\prime}=\sqrt{\hat{X}_{t}^{\prime}\left(1-\hat{X}_{t}^{\prime}\right)} d \tilde{W}_{t}^{\prime}, \quad \hat{X}_{0}^{\prime}=X_{0}$. We define for $t<T_{0+}^{N}$ the time changed $X_{t}^{\prime}=\hat{X}_{\tau^{-1}(t)}^{\prime}$, so that $\left(N, X^{\prime}\right)$ is solution to the SDE system (10) and hence, by uniqueness in law of the solution to this system, $\left(N, X^{\prime}\right)$ and $(N, X)$ have the same law. Since $\left(N, \hat{X}^{\prime}\right)$ and $(N, \hat{X})$ can be obtained as the same function of $\left(N, X^{\prime}\right)$ and $(N, X)$ respectively, we deduce that they share the same law up to time $T_{\max }$. Then we have

$$
\begin{aligned}
\mathbb{P} & \left(X_{t} \in(0,1) \forall t<T_{0+}^{N} \text { and } X_{T_{0+}^{N}-} \text { exists in }(0,1)\right) \\
& =\mathbb{P}\left(\hat{X}_{t} \in(0,1) \forall t<T_{\max } \text { and } \hat{X}_{T_{\max -}} \text { exists in }(0,1)\right) \\
& =\mathbb{P}\left(\hat{X}_{t}^{\prime} \in(0,1) \forall t<T_{\max } \text { and } \hat{X}_{T_{\max -}}^{\prime} \text { exists in }(0,1)\right)>0,
\end{aligned}
$$

since $N$ and $\hat{X}^{\prime}$ are independent and $\hat{X}^{\prime}$ is a Wright-Fisher diffusion. This concludes the proof, since $\left\{X_{t} \in(0,1), \forall t<T_{0+}^{N}\right.$ and $X_{T_{0+}^{N}-}$ exists in $\left.(0,1)\right\} \subset\left\{T_{0+}^{N}<T_{F}\right\}$, therefore $\mathbb{P}\left(T_{0+}^{N}<T_{F}\right)>0$.

Acknowledgements: This work was partially funded by the Chair "Modélisation Mathématique et Biodiversité" of VEOLIA-Ecole Polytechnique-MnHn-FX and also supported by public grants as part of the "Investissement d'avenir" project, reference ANR-11-LABX-0056-LMH, LabEx LMH, and reference ANR-10-CAMP-0151-02, FMJH, and by the Mission for Interdisciplinarity at the CNRS. 


\section{A Derivation of the generator (1) from an individual-based model}

\section{A.1 The model}

We consider a population of diploid hermaphroditic organisms, characterized by their genotype at one locus. There exist $L$ versions (alleles) of the gene at this locus and we denote by $1,2, . ., L$, these alleles. Individuals can then have genotype $i j$ for all $i$ and $j$ in $\llbracket 1, L \rrbracket$ (genotypes $i j$ and $j i$ are not distinguished), and we study the dynamics of the respective numbers of individuals with each genotype. We introduce a scaling parameter $K \in \mathbb{N} \backslash\{0\}$ that scales the initial population size and goes to infinity. The population is then represented at any time $t \geq 0$ by a symmetric positive matrix with size $L$, whose coefficients belong to $\mathbb{Z}_{+} / 2 K$ :

$$
\mathbf{N}^{K}(t)=\left(n_{i j}^{K}(t)\right)_{1 \leq i, j \leq L}
$$

where for all $i \in \llbracket 1, L \rrbracket, n_{i i}^{K}(t) \in \mathbb{Z}_{+} / K$ is the number of individuals with genotype $i i$ at time $t$, divided by $K$ and for all $i \neq j \in \llbracket 1, L \rrbracket, n_{i j}^{K}(t)+n_{j i}^{K}(t)=2 n_{i j}^{K}(t) \in \mathbb{Z}_{+} / K$ is the number of individuals with genotype $i j$ at time $t$, divided by $K$. For any time $t$, and for all $K, \mathbf{N}^{K}(t)$ belongs to the space $\mathcal{S}^{L}([0,+\infty))$ of symmetric matrices with positive real-valued coefficients.

Notation A.1. For any matrix $\nu=\left(\nu_{i j}\right)_{1 \leq i, j \leq L} \in \mathcal{S}^{L}([0,+\infty))$, we define $\nu_{\{i i\}}=\nu_{i i}$ and $\nu_{\{i j\}}=2 \nu_{i j}$ for all $i \neq j$.

We assume that the population follows a non-linear birth-and-death process with Mendelian reproduction and competition whose jump rates will be given later.

The following quantities play a main role in this study:

- $N^{K}(t)=\sum_{i, j \in \llbracket 1, L \rrbracket} n_{i j}^{K}(t)$ is the rescaled population size at time $t$,

- $n_{i}^{K}(t)=2 \sum_{j=1}^{L} n_{i j}^{K}(t)$ is the rescaled number of occurrences of allele $i$ at time $t$,

- $x_{i}^{K}(t)=\frac{n_{i}^{K}(t)}{2 N^{K}(t)}=\frac{\sum_{j} n_{i j}^{K}(t)}{\sum_{i, j} n_{i j}^{K}(t)}$ is the proportion of alleles $i$ at time $t$

- $x_{i j}^{K}(t)=\frac{n_{\{i j\}}^{K}(t)}{N^{K}(t)}$ is the proportion of genotypes $i j$ at time $t$,

- $\epsilon_{i j}^{K}(t)=x_{i}^{K}(t) x_{j}^{K}(t)-\frac{x_{i j}^{K}(t)}{2}$ is called the deviation of the population from Hardy-Weinberg structure, for genotype $i j$ with $i \neq j$.

For all $\mathbf{n}=\left(n_{i j}\right)_{i, j \in \llbracket 1, L \rrbracket} \in \mathcal{S}^{L}([0,+\infty)) \backslash \mathbf{0}$, we set for all $i \neq j$,

$$
\psi_{i j}(\mathbf{n})=\epsilon_{i j}=\frac{\left(\sum_{k} n_{i k}\right)\left(\sum_{l} n_{j l}\right)}{\left(\sum_{i, j} n_{i j}\right)^{2}}-\frac{n_{i j}}{\sum_{i, j} n_{i j}} .
$$

We obtain the following result:

Lemma A.2. For all $\mathbf{n}=\left(n_{i j}\right)_{i, j \in \llbracket 1, L \rrbracket} \in \mathcal{S}^{L}([0,+\infty)) \backslash \mathbf{0}$, let us define

$$
\begin{aligned}
\phi_{1}(\mathbf{n}) & =\sum_{i, j=1}^{L} n_{i j} ; \phi_{i}(\mathbf{n})=\frac{\sum_{j} n_{i j}}{\sum_{i, j} n_{i j}} \quad \text { for all } i \in \llbracket 2, L \rrbracket, \\
\left(\phi_{L+1}(\mathbf{n}), \ldots, \phi_{L(L+1) / 2}(\mathbf{n})\right) & =\left(\left(\psi_{1 j}(\mathbf{n})\right)_{1<j \leq L},\left(\psi_{2 j}(\mathbf{n})\right)_{2<j \leq L}, \ldots, \psi_{(L-1) L}(\mathbf{n})\right)
\end{aligned}
$$

The function

$$
\begin{aligned}
\phi: \mathcal{S}^{L}([0,+\infty)) \backslash \mathbf{0} & \rightarrow \phi\left(\mathcal{S}^{L}([0,+\infty)) \backslash \mathbf{0}\right) \\
\mathbf{n} & \mapsto \phi(\mathbf{n})=\left(\phi_{1}(\mathbf{n}), \ldots, \phi_{\frac{L(L+1)}{2}}(\mathbf{n})\right)
\end{aligned}
$$

is a bijection. 
Proof. Setting $x_{1}=1-x_{2}-x_{3}-\ldots-x_{L}$, we get that

$$
\left(n, x_{2}, x_{3}, \ldots, x_{L},\left(\epsilon_{1 j}\right)_{1 \leq i<j \leq L},\left(\epsilon_{2 j}\right)_{2 \leq i<j \leq L}, \ldots, \epsilon_{(L-1) L}\right)=\phi(\mathbf{x})
$$

if and only if

$$
\begin{aligned}
& n_{i j}=n\left(x_{i} x_{j}-\epsilon_{i j}\right) \quad \text { for all } i \neq j, \text { and } \\
& n_{i i}=n\left(x_{i}\right)^{2}+\sum_{j \neq i} \epsilon_{i j}, \quad \text { which gives the result. }
\end{aligned}
$$

For all $i, j \in \llbracket 1, L \rrbracket$, we now denote by $e_{i j}$ the square matrix with size $L$ such that for all $k, l \in \llbracket 1, L \rrbracket$, $e_{i j}(k, l)=\frac{\delta_{(i, j)}^{(k, l)}+\delta_{(j, i)}^{(k, l)}}{2}$. Individuals experience panmictic Mendelian reproduction. Therefore, for all $i<j \in \llbracket 1, L \rrbracket$, as long as the total population size $\sum_{1 \leq i, j \leq L} n_{i j}=n \neq 0$, the rate $\lambda_{i j}^{K}(\mathbf{n})\left(\operatorname{resp} . \lambda_{i i}^{K}(\mathbf{n})\right)$ at which the stochastic process $\mathbf{N}^{K}$ jumps from $\mathbf{n}=\left(n_{i j}\right)_{i, j \in \llbracket 1, L \rrbracket} \in \mathcal{S}^{L}([0,+\infty))$ to $\mathbf{n}+e_{i j} / K$ (resp. $\left.\mathbf{n}+e_{i i} / K\right)$ is given by:

$$
\begin{aligned}
\lambda_{i j}^{K}(\mathbf{x}) & =2 K b_{i j}^{K} n x_{i} x_{j} \\
\lambda_{i i}^{K}(\mathbf{x}) & =K b_{i i}^{K} n x_{i}^{2},
\end{aligned}
$$

where $b_{i j}^{K} \in[0,+\infty)$ for all $i \leq j \in \llbracket 1, L \rrbracket$. These birth rates are naturally all equal to 0 if $n=0$.

Each individual can die either naturally or due to the competition with other individuals. More precisely, for all $i \leq j \in \llbracket 1, L \rrbracket$, the rate $\mu_{i j}^{K}(\mathbf{x})$ at which the stochastic process $\mathbf{X}^{K}$ jumps from $\mathbf{x}=$ $\left(x_{i j}\right)_{i, j \in \llbracket 1, L \rrbracket} \in \mathcal{S}^{L}([0,+\infty))$ to $\mathbf{x}-e_{i j} / K$, is given by

$$
\mu_{i j}^{K}(\mathbf{x})=K\left(d_{i j}^{K}+K \sum_{1 \leq k, l \leq L} c_{i j, k l}^{K} x_{k l}\right) x_{\{i j\}}
$$

where $d_{i j}^{K} \in[0,+\infty)$ is the intrinsic death rate of an an individual with genotype $i j$, and $\left.\left.c_{i j, k l}^{K} \in\right] 0,+\infty\right)$ is the rate at which a given individual with genotype $i j$ dies due to the competition with a given individual with genotype $k l$ (we have used Notation A.1). We obviously assume that $c_{i j, k l}^{K}=c_{i j, l k}^{K}=c_{j i, k l}^{K}$ for all $i$, $j, k$, and $l$, since the two genotypes $i j$ and $j i$ are indistinguishable.

Note that for all $K \in \mathbb{N} \backslash\{0\}$, the pure jump process $\mathbf{X}^{K}$ is well-defined for all time $t \in[0,+\infty)$. Indeed, the process $\left(N^{K}(t), t \geq 0\right)$ is stochastically dominated by a logistic birth-and-death process $\bar{N}^{K}$ with birth, intrinsic death and competition parameters respectively equal to $\sup _{i, j} b_{i j}^{K}<+\infty$, inf $d_{i, j}^{K}$ and $\inf _{i, j, k, l} c_{k l, i j}^{K}>0$, which, from Chapter 8 of [1], does not explode, almost surely.

The stochastic process $\left(\mathbf{X}^{K}(t), t \geq 0\right)$ is therefore a pure jump process with values in $\mathcal{S}^{L}\left(\mathbb{R}_{+}\right)$(endowed with the distance $r$ such that $r(\mathbf{x}, \mathbf{y})=\max _{i, j}\left|x_{i j}-y_{i j}\right|$, for instance), absorbed at $\mathbf{0}$, and defined for all $t \geq 0$ by

$$
\mathbf{X}_{t}^{K}=\mathbf{X}_{0}^{K}+\sum_{1 \leq i \leq j \leq L}\left[\int_{0}^{t} \frac{e_{i j}}{K} \mathbf{1}_{\left\{\theta \leq \lambda_{i j}^{K}\left(\mathbf{X}_{s^{-}}^{K}\right)\right\}} \eta_{1}^{i j}(d s, d \theta)-\int_{0}^{t} \frac{e_{i j}}{K} \mathbf{1}_{\left\{\theta \leq \mu_{i j}^{K}\left(\mathbf{X}_{s^{-}}^{K}\right)\right\}} \eta_{2}^{i j}(d s, d \theta)\right]
$$

where the measures $\eta_{k}^{i j}$ for $i \leq j \in \llbracket 1, L \rrbracket$ and $k \in\{1,2\}$ are independent Poisson point measures on $[0,+\infty)^{2}$, with intensity $d s d \theta$. For all $K$, the law of $\mathbf{X}^{K}$ is then a probability measure on the space of trajectories $\mathbb{D}\left([0,+\infty), \mathcal{S}^{L}([0,+\infty))\right)$ which is the space of càd-làg functions, from $[0,+\infty)$ to $\mathcal{S}^{L}([0,+\infty))$, endowed with the Skorokhod topology. The extended generator $\mathcal{L}^{K}$ of $\left(\mathbf{X}^{K}(t), t \geq 0\right)$ satisfies, for all measurable function $f$ from $\mathcal{S}^{L}([0,+\infty))$ to $\mathbb{R}$, and for all $\mathbf{x} \in \mathcal{S}^{L}([0,+\infty))$ :

$$
\mathcal{L}^{K} f(\mathbf{x})=\sum_{1 \leq i \leq j \leq L}\left[\lambda_{i j}^{K}(\mathbf{x})\left(f\left(\mathbf{x}+\frac{e_{i j}}{K}\right)-f(\mathbf{x})\right)+\mu_{i j}^{K}(\mathbf{x})\left(f\left(\mathbf{x}-\frac{e_{i j}}{K}\right)-f(\mathbf{x})\right)\right],
$$

where the rates $\lambda_{i j}^{K}(\mathbf{x})$ and $\mu_{i j}^{K}(\mathbf{x})$ have been defined in Equations (12) and (13) for all $i \leq j$. 


\section{A.2 Slow-fast dynamics}

We now study the convergence of the sequence of stochastic processes $\left(\mathbf{X}^{K}(t), t \geq 0\right)_{K \in \mathbb{N} \backslash\{0\}}$ toward a slow-fast stochastic diffusion dynamic, as done in [8]. To this aim, demographic parameters must be properly rescaled, according to the following assumptions, for $\gamma>0$ :

$$
\left.\left.\left.\left.b_{i j}^{K}=\gamma K+\beta_{i j} \in\right] 0,+\infty\right), \quad d_{i j}^{K}=\gamma K+\delta_{i j} \in[0,+\infty), \quad \text { and } \quad c_{i j, k l}^{K}=\frac{\alpha_{i j, k l}}{K} \in\right] 0,+\infty\right) .
$$

Besides, we assume that

$$
\text { there exists a constant } C<\infty \text { such that } \sup _{K} \mathbb{E}\left(\left(N^{K}(0)\right)^{3}\right) \leq C \text {. }
$$

Then, from Lemma 1 of [6] and the proof of Theorem 5.3 of [14]:

(i) There exists a constant $C>0$ such that

$$
\sup _{K} \sup _{t \geq 0} \mathbb{E}\left(\left(N^{K}(t)\right)^{3}\right) \leq C .
$$

(ii) For all $T<+\infty$, there exists a constant $C_{T}$ such that

$$
\sup _{K} \mathbb{E}\left(\sup _{t \leq T}\left(N^{K}(t)\right)^{3}\right) \leq C_{T}
$$

The following proposition gives the convergence of the fast variables $\left(\left(\epsilon_{i j}^{K}(t)\right)_{1 \leq i<j \leq L}, t \geq 0\right)$ toward 0 and is an extension of Proposition 3.2 of [8] for a larger number of alleles. The proof of this result can be found in [9], Chapter 4, Appendix A.

Proposition A.3. Under the Hypothesis (15), for all times $s, t>0$ and for all $i \neq j \in \llbracket 1, L \rrbracket$, $\sup _{t \leq u \leq t+s} \mathbb{E}\left(\left(\epsilon_{i j}^{K}(u)\right)^{2}\right) \rightarrow 0$ when $K$ goes to infinity.

We next study the asymptotic behavior of the sequence of stochastic processes constituted of the remaining variables $\left(N^{K}(t), x_{2}^{K}(t), x_{3}^{K}(t), \ldots, x_{L}^{K}(t)\right)_{t \geq 0}$ introduced in Lemma A.2, when $K$ goes to infinity. For more simplicity, we first consider the sequence of stochastic processes $\left(\left(n_{1}^{K}(t), n_{2}^{K}(t), \ldots, n_{L}^{K}(t)\right)_{t \geq 0}\right)_{K \in \mathbb{N} \backslash\{0\}}$ giving the respective numbers of occurrences of the different alleles, whose dynamics are simpler. The proof of the following can be found in [9], Chapter 4, Appendix A and is a generalization of the proof of Theorem 1 in [8].

Theorem A.4. Under (15), if the sequence $\left(n_{1}^{K}(0), n_{2}^{K}(0), \ldots, n_{L}^{K}(0)\right)_{K \in \mathbb{N} \backslash\{0\}}$ converges in law toward a random variable $\left(n_{1}(0), n_{2}(0), \ldots, n_{L}(0)\right) \in[0,+\infty)^{L}$ when $K$ goes to infinity, then for all $T>0$, the sequence of stochastic processes $\left(\left(n_{1}^{K}(t), n_{2}^{K}(t), \ldots, n_{L}^{K}(t)\right), t \in[0, T]\right)$ converges in law in $\mathbb{D}\left([0, T],[0,+\infty)^{L}\right)$ when $K$ goes to infinity, toward a time-continuous diffusion process $\left(\left(n_{1}(t), n_{2}(t), \ldots, n_{L}(t)\right), t \in[0, T]\right)$ starting from $\left(n_{1}(0), n_{2}(0), \ldots, n_{L}(0)\right)$, which is the unique continuous solution of the martingale problem:

$$
g\left(n_{1}(t), n_{2}(t), \ldots, n_{L}(t)\right)-g\left(n_{1}(0), n_{2}(0), \ldots, n_{L}(0)\right)-\int_{0}^{t} \mathcal{L} g\left(n_{1}(s), n_{2}(s), \ldots, n_{L}(s)\right) d s
$$

is a martingale for all function $g \in \mathcal{C}_{b}^{2}\left([0,+\infty)^{L}, \mathbb{R}\right)$ where $\mathcal{L}$ satisfies

$$
\begin{aligned}
\mathcal{L} g\left(n_{1}, \ldots, n_{L}\right) & =\sum_{i=1}^{L} \frac{\partial g}{\partial n_{i}}(n)\left[\sum_{j=1}^{L}\left(\beta_{i j}-\delta_{i j}-\sum_{k, l} \alpha_{i j, k l} \frac{n_{k} n_{l}}{2 \sum_{k} n_{k}}\right) \frac{n_{i} n_{j}}{\sum_{k} n_{k}}\right] \\
& +\gamma \sum_{i=1}^{L} \frac{\partial^{2} g}{\partial n_{i}^{2}}(n)\left[\frac{\left(n_{i}\right)^{2}}{\sum_{k} n_{k}}+n_{i}\right]+\gamma \sum_{i<j} \frac{\partial^{2} g}{\partial n_{i} \partial n_{j}}(n)\left[\frac{2 n_{i} n_{j}}{\sum_{k} n_{k}}\right]
\end{aligned}
$$

for all point $n=\left(n_{1}, \ldots, n_{L}\right)$ of $[0,+\infty)^{L}$. 
Note that the diffusion coefficients of the generator $\mathcal{L}$ go to 0 when the total $\sum_{k} n_{k}$ goes to 0 . The system of equations (16) and (17) admits a unique strong solution up to time $T_{\epsilon}=\inf \left\{t>0, n_{1}(t)+\right.$ $\left.n_{2}(t)+\ldots+n_{L}(t) \geq \epsilon\right\}$. Then from Theorem 6.2, Chapter 4 of [12], it admits a unique strong solution up to time $T_{0+}=\lim _{\epsilon \rightarrow 0} T_{\epsilon}$.

From Theorem A.4, we deduce for all $\epsilon>0$ the convergence of the sequence of stochastic processes $\left(N^{K}(t), x_{2}^{K}(t), x_{3}^{K}(t), \ldots x_{L}^{K}(t)\right)_{t \geq 0}$ stopped when $N^{K}(t) \leq \epsilon$, toward a $L$-dimensional diffusion process $\left(N(),. x_{2}(),. \ldots, x_{L}().\right) . \wedge T_{\epsilon}$, stopped when $N(t) \leq \epsilon$ :

Corollary A.5. For all $\epsilon>0$ and $T>0$, let us define $T_{\epsilon}^{K}=\inf \left\{t \in[0, T]: N^{K}(t) \leq \epsilon\right\}$. If the sequence of random variables $\left(N^{K}(0), x_{2}^{K}(0), x_{3}^{K}(0), \ldots x_{L}^{K}(0)\right) \in\left[\epsilon,+\infty\left[\times[0,1]^{L-1}\right.\right.$ converges in law when $K$ goes to infinity, toward a random vector $\left.\left(N(0), x_{2}(0), x_{3}(0), \ldots x_{L}(0)\right) \in\right] \epsilon,+\infty\left[\times[0,1]^{L-1}\right.$, then the sequence of stopped stochastic processes $\left\{\left(N^{K}\left(t \wedge T_{\epsilon}^{K}\right), x_{2}^{K}\left(t \wedge T_{\epsilon}^{K}\right), x_{3}^{K}\left(t \wedge T_{\epsilon}^{K}\right), \ldots, x_{L}^{K}\left(t \wedge T_{\epsilon}^{K}\right)\right)_{0 \leq t \leq T}\right\}_{K \geq 1}$ converges in law in $\mathbb{D}\left([0, T],\left[\epsilon, \infty\left[\times[0,1]^{L-1}\right)\right.\right.$ when $K$ goes to infinity, toward a continuous diffusion process $\left(N\left(t \wedge T_{\epsilon}\right), x_{2}\left(t \wedge T_{\epsilon}\right), \ldots, x_{L}\left(t \wedge T_{\epsilon}\right)\right)_{0 \leq t \leq T}$ stopped at time $T_{\epsilon}=\inf \left\{t \in[0, T]: N_{t}=\epsilon\right\}$, starting from $\left(N(0), x_{2}(0), x_{3}(0), \ldots x_{L}(0)\right)$ and whose infinitesimal generator $\mathcal{L}_{1}$ is defined for all function $f \in$ $\mathcal{C}_{b}^{2}\left(\left[\epsilon, \infty\left[\times[0,1]^{L-1}, \mathbb{R}\right)\right.\right.$ by

$$
\begin{aligned}
\mathcal{L}_{1} f\left(n, x_{2}, \ldots, x_{L}\right)=n\left(\sum_{1 \leq i, j \leq L}\left(\beta_{i j}-\delta_{i j}-\sum_{1 \leq k, l \leq L} \alpha_{i j, k l} n x_{k} x_{l}\right) x_{i} x_{j}\right) \frac{\partial f}{\partial n}\left(n, x_{2}, \ldots, x_{L}\right) \\
+\gamma n \frac{\partial^{2} f}{\partial n^{2}}\left(n, x_{2}, \ldots, x_{L}\right) \\
+\sum_{i=2}^{L}\left[x _ { i } \sum _ { j = 1 } ^ { L } \sum _ { k = 1 } ^ { L } x _ { j } x _ { k } \left(\left(\beta_{i k}-\beta_{j k}\right)-\left(\delta_{i k}-\delta_{j k}\right)\right.\right. \\
\left.\left.\quad-\sum_{1 \leq l, m \leq L}\left(\alpha_{i k, m l}-\alpha_{j k, m l}\right) n x_{m} x_{l}\right)\right] \frac{\partial f}{\partial x_{i}}\left(n, x_{2}, \ldots, x_{L}\right) \\
+\sum_{i=2}^{L} \gamma \frac{x_{i}\left(1-x_{i}\right)}{2 n} \frac{\partial^{2} f}{\partial x_{i}^{2}}\left(n, x_{2}, \ldots, x_{L}\right)-\sum_{i \neq j \in \llbracket 2, N \rrbracket} \gamma \frac{x_{i} x_{j}}{2 n} \frac{\partial^{2} f}{\partial x_{i} \partial x_{j}}\left(n, x_{2}, \ldots, x_{L}\right)
\end{aligned}
$$

The link with the generator (1) can be seen by setting $\rho=\beta_{11}-\delta_{11}, s_{i j}=\left(\beta_{i j}-\delta_{i j}\right)-\left(\beta_{11}-\delta_{11}\right)$, $\alpha=\alpha_{11,11}$ and $c_{i j, k l}=\alpha_{i j, k l}-\alpha_{11,11}$.

\section{B Integrability properties for diffusion processes}

Proofs of Theorems 2.1, Lemmas 2.2, 2.3 and Theorem 3.1 rely on the integrability of paths of diffusion processes. This section is devoted to the statement and the proof of a criterion for such integrability (Theorem B.2). More precisely, this result states that, depending on the behavior of the diffusion and drift coefficients near absorption, the integral of the paths of diffusion processes are either almost surely finite or almost surely infinite. This $0-1$ law criterion has already been proved by various methods, using a combination of the local time formula and Ray-Knight Theorem [11, 21, 19] (see also [10, 13] for proofs in particular settings). We give a simpler proof of this criterion, which also provides explicit bounds for the moments of perpetual integrals and can be easily extended to more general one dimensional Markov processes. Then, we extend this result to a diffusion taking values in a compact subset and finally to non-homogeneous processes by the use of Girsanov's transform.

\section{B.1 General diffusion processes on $[0,+\infty)$}

Let us consider a general one-dimensional diffusion process $\left(Z_{t}, t \geq 0\right)$ (that is a continuous strong Markov process) with values in $[0,+\infty)$. We denote by $T_{z}$ the hitting time of $z \in[0,+\infty)$ by the process $Z$ :

$$
T_{z}=\inf \left\{t \geq 0, Z_{t}=z\right\} .
$$

When the process $Z$ has to be specified, this time will be denoted $T_{z}^{Z}$. 
Let us denote by $\mathbb{P}_{z}$ the law of $Z$ starting from $z$. We assume that $Z$ is regular $(\forall z \in(0,+\infty), \forall y \in$ $\left.(0,+\infty), \mathbb{P}_{z}\left(T_{y}<+\infty\right)>0\right)$. This implies that for any $a<b \in(0,+\infty)$ and $a \leq z \leq b, \mathbb{E}_{z}\left(T_{a} \wedge T_{b}\right)<+\infty$ and we can associate with $Z$ a scale function $s$ and a locally finite speed measure $m$ on $[0,+\infty$ ) (see [22, Chapter VII]). We moreover assume that for all $z \in(0,+\infty)$,

$$
\mathbb{P}_{z}\left(T_{0}=T_{0} \wedge T_{e}<+\infty\right)=1
$$

where $T_{e}$ is the explosion time.

Lemma B.1. Condition (18) is equivalent to

$$
s(+\infty)=+\infty \quad ; \quad s(0)>-\infty \quad ; \quad \int_{0+}(s(y)-s(0)) m(d y)<+\infty .
$$

Note that Condition (19) is well known in the case where $Z$ is solution of a stochastic differential equation (cf. [18] p.348, [16] p.450).

Proof. Assume first that (18) is satisfied. As $Z$ has scale $s, s(Z)$ is a local martingale on $(s(0), s(+\infty))$ such that $T_{s(0)}^{s(Z)}<T_{s(+\infty)}^{s(Z)}$ a.s.. We deduce that $s(0)>-\infty$ and $s(+\infty)=+\infty$. The diffusion $s(Z)$ has a natural scale with speed measure $\tilde{m}=m \circ s^{-1}$ (see [22], Chapter VII). Since it attains $s(0)$ in finite time almost surely, we deduce using [23, Theorem 51-2] that $\int_{s(0)+}(u-s(0)) \tilde{m}(d u)<+\infty$. As $\int_{s(0)+}(u-s(0)) \tilde{m}(d u)<+\infty \Longleftrightarrow \int_{0+}(s(y)-s(0)) m(d y)<+\infty$, we obtain (19). Conversely, assume (19). Conditions $s(0)>-\infty$ and $s(+\infty)=+\infty$ imply that the local martingale $s(Z)$ doesn't explode a.s.. Since $\int_{0+}(s(y)-s(0)) m(d y)<+\infty$, then $\int_{s(0)+}(u-s(0)) \tilde{m}(d u)<+\infty$ and the process $s(Z)$ attains $s(0)$ in finite time a.s., so does the process $Z$.

Since the function $s$ is defined up to a constant, we choose by convention $s(0)=0$ as soon as $s(0)>-\infty$.

The following theorem gives a $0-1$ law criterion for the finiteness/infiniteness of perpetual integrals of diffusion processes, for which we provide a new and simple proof.

Theorem B.2. Let $\left(Z_{t}, t \geq 0\right)$ be a regular diffusion process on $[0,+\infty)$ with scale function $s$ and speed measure $m$ on $(0,+\infty)$ satisfying (19). Let also $f$ be a non-negative locally integrable function on $(0,+\infty)$. Then, for all $z>0$ and all $n \geq 1$,

$$
\mathbb{E}_{z}\left[\left(\int_{0}^{T_{0}} f\left(Z_{s}\right) d s\right)^{n}\right] \leq n !\left(\int_{0}^{\infty} s(y) f(y) m(d y)\right)^{n}
$$

and

$$
\begin{aligned}
& \int_{0^{+}} s(y) f(y) m(d y)<+\infty \Longleftrightarrow \int_{0}^{T_{0}} f\left(Z_{s}\right) d s<+\infty \quad \mathbb{P}_{z} \text { - almost surely } \\
& \int_{0^{+}} s(y) f(y) m(d y)=+\infty \Longleftrightarrow \int_{0}^{T_{0}} f\left(Z_{s}\right) d s=+\infty \quad \mathbb{P}_{z} \text { - almost surely. }
\end{aligned}
$$

Proof. Because of the non-explosion assumption (19), we have $\int_{0}^{T_{0}} f\left(Z_{s}\right) d s<+\infty \Leftrightarrow \forall k \in \mathbb{N}, \int_{0}^{T_{0}} f\left(Z_{s}\right) \mathbf{1}_{Z_{s} \leq k} d s<$ $+\infty$ and $\int_{0}^{T_{0}} f\left(Z_{s}\right) d s=+\infty \Leftrightarrow \exists k \in \mathbb{N}$ such that $\int_{0}^{T_{0}} f\left(Z_{s}\right) \mathbf{1}_{Z_{s} \leq k} d s=+\infty$. Hence it is sufficient to prove Theorem B.4 for functions $f$ satisfying $\int_{a}^{\infty} f(x) s(x) m(d x)<+\infty$ for all $a>0$. We make this assumption from the rest of the proof.

As $Z$ has scale function $s$ and speed measure $m$, the process $s(Z)$ is on a natural scale with speed measure $m \circ s^{-1}$. Then it is enough to prove the result for $Z$ on a natural scale. In particular, we have the following Green formula (see [Chapter 23] of [17])

$$
\mathbb{E}_{x}\left(\int_{0}^{T_{0}} f\left(Z_{s}\right) d s\right)=\int_{(0,+\infty)} 2(x \wedge y) f(y) m(d y)
$$


Noting that

$$
\int_{0}^{T_{0}} f\left(Z_{s}\right) d s=\sum_{k=1}^{\infty} \int_{T_{x / k}}^{T_{x /(k+1)}} f\left(Z_{s}\right) d s
$$

one easily checks that, under $\mathbb{P}_{x}$ for any $x \in(0,+\infty), \int_{0}^{T_{0}} f\left(Z_{s}\right) d s<+\infty$ satisfies a $0-1$ law. Indeed, the random variables $\int_{T_{x / k}}^{T_{x /(k+1)}} f\left(Z_{s}\right) d s, k \geq 1$ are non-negative and independent (strong Markov property) and almost surely finite because of our assumptions and the Green's formula applied under $\mathbb{P}_{x / k}$ up to time $T_{x / k+1}$. Hence the above series is finite with probability zero or one.

Let us now assume that $\int_{(0,+\infty)} y f(y) m(d y)<+\infty$. Then $\int_{0}^{T_{0}} f\left(Z_{s}\right) d s<\infty$ almost surely and, for all $n \geq 1$,

$$
\begin{aligned}
\mathbb{E}_{x}\left[\left(\int_{0}^{T_{0}} f\left(Z_{s}\right) d s\right)^{n}\right] & =\mathbb{E}_{x}\left[n \int_{0}^{T_{0}} f\left(Z_{s}\right)\left(\int_{s}^{T_{0}} f\left(Z_{u}\right) d u\right)^{n-1} d s\right] \\
& =n \int_{0}^{\infty} \mathbb{E}_{x}\left[\mathbf{1}_{s<T_{0}} f\left(Z_{s}\right)\left(\int_{s}^{T_{0}} f\left(Z_{u}\right) d u\right)^{n-1}\right] d s \\
& =n \mathbb{E}_{x}\left[\int_{0}^{T_{0}} f\left(Z_{s}\right) \mathbb{E}_{Z_{s}}\left(\left(\int_{0}^{T_{0}} f\left(Z_{u}\right) d u\right)^{n-1}\right) d s\right],
\end{aligned}
$$

where we used the Markov property. We immediately deduce by induction that

$$
\mathbb{E}_{x}\left[\left(\int_{0}^{T_{0}} f\left(Z_{s}\right) d s\right)^{n}\right] \leq n !\left(\int_{(0,+\infty)} 2 y f(y) m(d y)\right)^{n} .
$$

This concludes the proof of the first part of Theorem B.2 (the inequality is trivial when $\int_{(0,+\infty)} y f(y) m(d y)=$ $+\infty)$.

Assume now that $\int_{(0,+\infty)} y f(y) m(d y)=+\infty$ and fix $x \in(0,+\infty)$. For all $k \geq 1$, we set

$$
f_{k}(y)= \begin{cases}f(y) & \text { if } y \geq 1 \\ f(y) \wedge k & \text { if } y<1\end{cases}
$$

In particular, $\int_{(0,+\infty)} f_{k}(y) y m(d y)<\infty$ for all $k \geq 1$ and hence, using the inequalities established above and then the fact that $\int_{(0,+\infty)} 2 y f_{k}(y) m(d y)$ goes to infinity and the fact that $y f(y) m(d y)$ is assumed to be finite on neighborhood of $+\infty$, we deduce that for $k$ large enough

$$
\begin{aligned}
\mathbb{E}_{x}\left[\left(\int_{0}^{T_{0}} f_{k}\left(Z_{s}\right) d s\right)^{2}\right] \leq 2\left(\int_{(0,+\infty)} 2 y f_{k}(y) m(d y)\right)^{2} \\
\leq 2\left(\int_{(0,+\infty)} 2(y \wedge x) f_{k}(y) m(d y)+\int_{x}^{\infty} 2(y-x) f(y) m(d y)\right)^{2} \\
\leq 4\left(\int_{(0,+\infty)} 2(y \wedge x) f_{k}(y) m(d y)\right)^{2}+4\left(\int_{x}^{\infty} 2(y-x) f(y) m(d y)\right)^{2} \\
\leq 5\left(\int_{(0,+\infty)} 2(y \wedge x) f_{k}(y) m(d y)\right)^{2} \leq 5\left[\mathbb{E}_{x}\left(\int_{0}^{T_{0}} f_{k}\left(Z_{s}\right) d s\right)\right]^{2}
\end{aligned}
$$


We deduce that, for $k$ large enough,

$$
\mathbb{P}_{x}\left(\int_{0}^{T_{0}} f_{k}\left(Z_{s}\right) d s \geq \frac{\mathbb{E}_{x}\left(\int_{0}^{T_{0}} f_{k}\left(Z_{s}\right) d s\right)}{2}\right) \geq \frac{1}{20} .
$$

Indeed, for any random variable $Y \geq 0$ such that $\mathbb{E}\left(Y^{2}\right) \leq 5 \mathbb{E}(Y)^{2}$, we have, setting $M=\mathbb{E}(Y)$,

$$
\begin{aligned}
5 M^{2} & \geq \mathbb{E}\left(Y^{2}\right) \geq \mathbb{E}\left(Y^{2} \mid Y \geq M / 2\right) \mathbb{P}(Y \geq M / 2) \geq \mathbb{E}(Y \mid Y \geq M / 2)^{2} \mathbb{P}(Y \geq M / 2) \\
& \geq \frac{\mathbb{E}\left(Y \mathbf{1}_{Y \geq M / 2}\right)^{2}}{\mathbb{P}(Y \geq M / 2)} \geq \frac{M^{2} / 4}{\mathbb{P}(Y \geq M / 2)}
\end{aligned}
$$

and hence $\mathbb{P}(Y \geq M / 2) \geq 1 / 20$. Now using the fact that $f_{k}$ is increasing in $k$, we deduce that, for $k$ large enough,

$$
\mathbb{P}_{x}\left(\int_{0}^{T_{0}} f\left(Z_{s}\right) d s \geq \frac{\mathbb{E}_{x}\left(\int_{0}^{T_{0}} f_{k}\left(Z_{s}\right) d s\right)}{2}\right) \geq 1 / 20 .
$$

Since $\mathbb{E}_{x}\left(\int_{0}^{T_{0}} f_{k}\left(Z_{s}\right) d s\right)$ is not bounded in $k$, we deduce that $\mathbb{P}_{x}\left(\int_{0}^{T_{0}} f\left(Z_{s}\right) d s=+\infty\right) \geq 1 / 20$. This and the fact that $\left\{\int_{0}^{T_{0}} f\left(Z_{s}\right) d s=+\infty\right\}$ satisfies a $0-1$ law conclude the proof.

The equivalences stated in Theorem B.2 are particularly useful when $Z$ is solution of

$$
d Z_{t}=\sigma\left(Z_{t}\right) d B_{t}+b\left(Z_{t}\right) d t \quad ; \quad Z_{0}>0,
$$

where $B$ is a one dimensional Brownian motion, and $\sigma:(0,+\infty) \rightarrow(0,+\infty)$ and $b:(0,+\infty) \rightarrow \mathbb{R}$ are measurable functions such that $b / \sigma^{2}$ is locally integrable. The scale function (up to a constant) and speed measure equal to

$$
s(x)=\int_{c}^{x} \exp \left(-2 \int_{c}^{y} \frac{b(z)}{\sigma^{2}(z)} d z\right) d y \quad ; \quad m(d x)=\frac{2 d x}{s^{\prime}(x) \sigma^{2}(x)},
$$

(cf. [17, Chapter 23]).

Corollary B.3. Assume that $Z$ is solution of $(20)$ with $s(+\infty)=+\infty$ and $\int_{0+} s(y) m(d y)<+\infty$. Let us consider a non negative locally integrable function $f$ on $(0,+\infty)$. Then, under $\mathbb{P}_{z}$,

$$
\begin{aligned}
\int_{0^{+}} \frac{f(y) s(y)}{s^{\prime}(y) \sigma^{2}(y)} d y=+\infty \quad \Longleftrightarrow \int_{0}^{T_{0}} f\left(Z_{s}\right) d s=+\infty \quad \text { almost surely, } \\
\int_{0^{+}} \frac{f(y) s(y)}{s^{\prime}(y) \sigma^{2}(y)} d y<+\infty \quad \Longleftrightarrow \quad \int_{0}^{T_{0}} f\left(Z_{s}\right) d s<+\infty \quad \text { almost surely. }
\end{aligned}
$$

Let us give two examples for population size processes.

Example 1. Branching process with immigration. Let us consider the solution of the stochastic differential equation $d N_{t}=\sigma \sqrt{N_{t}} d B_{t}+\beta d t, \beta>0$. Computing $s$ and $m$ as in (21), we easily obtain that (18) $\Longleftrightarrow$ $\beta / \sigma^{2}<1 / 2$. Applying Corollary B.3 with $f(y)=1 / y^{\alpha}$, we have

$$
\int_{0}^{T_{0}} \frac{1}{\left(N_{s}\right)^{\alpha}} d s=+\infty \quad \text { a.s. } \Longleftrightarrow \alpha \geq 1 ; \int_{0}^{T_{0}} \frac{1}{\left(N_{s}\right)^{\alpha}} d s<+\infty \quad \text { a.s. } \Longleftrightarrow \alpha<1 .
$$

In the particular case $\alpha=1$, the authors of [13] propose an other approach based on self-similarity properties. 
Example 2. Logistic diffusion process. Let us consider the process

$$
d N_{t}=\sqrt{N_{t}} d B_{t}+N_{t}\left(b-c N_{t}\right) d t ; N_{0}>0
$$

where $c>0$. Then $s(y)=\int_{0}^{y} e^{c z^{2}-2 b z} d z$ and $m(d y)=\frac{2 e^{-c y^{2}+2 b y}}{y} d y$ and $\int_{0^{+}} s(y) m(d y)<+\infty$, since $\frac{s(y)}{s^{\prime}(y) y} \rightarrow_{y \rightarrow 0}$ 1. (Note that if $c=0$, the condition $s(+\infty)=+\infty$ is not satisfied). It is immediate to check that (22) also holds.

\section{B.2 General diffusion processes on $(a, b)$}

Let us consider a general diffusion process $\left(X_{t}, t \geq 0\right)$ with scale function $s$ and locally finite speed measure $m$ on $(a, b)$, with $-\infty<a<b<+\infty$. Let us denote by $T_{a}$ and $T_{b}$ the hitting times of $a$ and $b$ respectively by the process $X$. We assume that, for all $x \in(a, b), \mathbb{P}_{x}\left(T_{a} \wedge T_{b}<+\infty\right)=1$. This is the case if and only if one of the following properties is satisfied

$(i)-\infty<s(a)<s(b)<+\infty ; \int_{a^{+}}(s(y)-s(a)) m(d y)<+\infty$ and $\int^{b^{-}}(s(b)-s(y) m(d y)<+\infty ;$

$(i i)-\infty<s(a)$ and $s(b)=+\infty ; \int_{a^{+}}(s(y)-s(a)) m(d y)<+\infty$;

(iii) $s(a)=-\infty$ and $s(b)<+\infty ; \int^{b^{-}}(s(b)-s(y)) m(d y)<+\infty$.

Theorem B.4. Fix $x \in(a, b)$ and let $f:(a, b) \rightarrow \mathbb{R}_{+}$be a locally bounded measurable function. Then

$$
\begin{gathered}
\int^{b^{-}}(s(b)-s(y)) f(y) m(d y)=\infty \Leftrightarrow \mathbb{P}_{x}\left(\left\{\int_{0}^{T_{b}} f\left(X_{s}\right) d s=\infty\right\} \cap\left\{T_{b}<T_{a}\right\}\right)=\mathbb{P}_{x}\left(T_{b}<T_{a}\right) \\
\int^{b^{-}}(s(b)-s(y)) f(y) m(d y)<\infty \Leftrightarrow \mathbb{P}_{x}\left(\left\{\int_{0}^{T_{b}} f\left(X_{s}\right) d s<\infty\right\} \cap\left\{T_{b}<T_{a}\right\}\right)=\mathbb{P}_{x}\left(T_{b}<T_{a}\right) .
\end{gathered}
$$

A similar result holds at the boundary $a$.

Proof. As in the proof of Theorem B.2, it is enough to prove the result in the case where $s$ is the identity function. Without loss of generality, we take $(a, b)=(0,1)$. Let us consider $x \in(0,1)$, fix $\varepsilon \in(0,1-x)$ and consider a locally finite measure $m^{\varepsilon}$ on $(0,+\infty)$ such that the restriction of $m^{\varepsilon}$ on $(0,1-\varepsilon)$ is equal to the restriction of $m$ on $(0,1-\varepsilon)$. Let $X^{\varepsilon}$ be a diffusion process on natural scale on $(0,+\infty)$ with speed measure $m^{\varepsilon}$ and starting from $x$, built as a time change of the same Brownian motion as $X$. Because of this construction, $X$ and $X^{\varepsilon}$ coincide up to time $T_{0}$ on the event $\left\{T_{0}<T_{1-\varepsilon}\right\}$.

Now, by Theorem B.2 applied to $X^{\varepsilon}$ and $f^{\varepsilon}: y \mapsto f(y) \mathbb{1}_{y \leq 1-\varepsilon}$, we deduce that

$$
\begin{aligned}
\int_{0}^{T_{0}} f\left(X_{s}^{\varepsilon}\right) \mathbb{1}_{X_{s}^{\varepsilon} \leq 1-\varepsilon} d s=+\infty & \text { almost surely } \Longleftrightarrow \int_{0^{+}} y f(y) m(d y)=+\infty \\
\int_{0}^{T_{0}} f\left(X_{s}^{\varepsilon}\right) \mathbb{1}_{X_{s}^{\varepsilon} \leq 1-\varepsilon} d s<+\infty & \text { almost surely } \Longleftrightarrow \int_{0^{+}} y f(y) m(d y)<+\infty .
\end{aligned}
$$

Since on the event $T_{0}<T_{1-\varepsilon}, X$ and $X^{\varepsilon}$ coincide up to time $T_{0}$ and $X_{s} \leq 1-\varepsilon$ holds for $s \leq T_{0}$, then up to $\mathbb{P}_{x}$-negligible events,

$$
\begin{array}{ccc}
\int_{0^{+}} y f(y) m(d y)=+\infty & \Longrightarrow \int_{0}^{T_{0}} f\left(X_{s}\right) d s=+\infty \quad \text { on } T_{0}<T_{1-\varepsilon} . \\
\int_{0^{+}} y f(y) m(d y)<+\infty & \Longrightarrow \int_{0}^{T_{0}} f\left(X_{s}\right) d s<+\infty \quad \text { on } T_{0}<T_{1-\varepsilon} .
\end{array}
$$


The continuity of the paths of $X$ implies that $\left\{T_{0}<T_{1}\right\}=\cup_{0<\varepsilon<1-x}\left\{T_{0}<T_{1-\varepsilon}\right\}$, which yields, up to negligible events,

$$
\begin{array}{ccc}
\int_{0^{+}} y f(y) m(d y)=+\infty & \Longrightarrow \int_{0}^{T_{0}} f\left(X_{s}\right) d s=+\infty \quad \text { on } T_{0}<T_{1} . \\
\int_{0^{+}} y f(y) m(d y)<+\infty & \Longrightarrow \quad \int_{0}^{T_{0}} f\left(X_{s}\right) d s<+\infty \quad \text { on } T_{0}<T_{1} .
\end{array}
$$

This concludes the proof of the direct implications in Theorem B.4.

Now, assume for instance that $\int_{0}^{T_{0}} f\left(X_{s}\right) d s=+\infty$ on $T_{0}<T_{1}$. Then, a fortiori, $\int_{0}^{T_{0}} f\left(X_{s}\right) d s=+\infty$ on $T_{0}<T_{1-\varepsilon}$ for any $\varepsilon \in(0,1-x)$. This implies that $\int_{0}^{T_{0}} f\left(X_{s}^{\varepsilon}\right) d s=+\infty$ on $T_{0}<T_{1-\varepsilon}$. But $T_{0}<T_{1-\varepsilon}$ happens with probability $x /(1-\varepsilon)>0$ by definition of the natural scale. We deduce from Theorem B.2 that $\int_{0^{+}} y f(y) m(d y)<+\infty$ does not hold and hence, because $f$ is non-negative, that $\int_{0^{+}} y f(y) m(d y)=+\infty$. This provides the first $\Leftarrow$ implication in Theorem B.4. The second $\Leftarrow$ implication in Theorem B.4 is proved using similar arguments.

The result at boundary $b$ is proved similarly.

\section{B.3 Extension to non-homogeneous processes by use of Girsanov transform}

We are interested in generalized one-dimensional stochastic differential equations of the form

$$
d X_{t}=\sigma\left(X_{t}\right) d B_{t}+b\left(X_{t}\right) d t+q\left(X_{t}, \theta_{t}\right) d t, X_{0}>0,
$$

where $\left(B_{t}, t \geq 0\right)$ is a Brownian motion for some filtration $\left(\mathcal{F}_{t}\right)_{t}$ and $\left(\theta_{t}, t \geq 0\right)$ is predictable with respect to $\left(\mathcal{F}_{t}\right)_{t}$. The process $\left(\theta_{t}\right)_{t}$ can for example model an environmental heterogeneity.

Assumption $(H)$ : We consider real functions $\sigma$ and $b$ such that for any Brownian motion $W$ on some probability space, the one-dimensional stochastic differential equation $d Z_{t}=\sigma\left(Z_{t}\right) d W_{t}+b\left(Z_{t}\right) d t, Z_{0}>0$ satisfies the assumptions of Corollary B.3.

Theorem B.5. Let us consider a solution $X$ of (23) where $\sigma$ and b satisfy Assumption $(H)$. We also assume that $T_{0}=T_{0}^{X}<+\infty$ almost surely and that the sequence $\left(T_{k}^{X}\right)_{k \in \mathbb{N}^{*}}$ tends almost surely to infinity as $k$ tends to infinity.

Next, we assume that for any $k \in \mathbb{N} \backslash\{0\}$,

$$
\mathbb{E}\left(\exp \left(\frac{1}{2} \int_{0}^{T_{k}^{X}} \frac{q^{2}\left(X_{s}, \theta_{s}\right)}{\sigma^{2}\left(X_{s}\right)} d s\right)\right)<+\infty .
$$

Let $f$ be a non negative locally bounded measurable function on $(0,+\infty)$. We have

$$
\begin{aligned}
\int_{0^{+}} f(y) s(y) m(d y)=+\infty & \Longleftrightarrow \int_{0}^{T_{0}^{X}} f\left(X_{s}\right) d s=+\infty \quad \text { almost surely, } \\
\int_{0^{+}} f(y) s(y) m(d y)<+\infty & \Longleftrightarrow \int_{0}^{T_{0}^{X}} f\left(X_{s}\right) d s<+\infty \quad \text { almost surely }
\end{aligned}
$$

where $s$ and $m$ are defined in (21).

Note that (24) holds true as soon as, for all $k \in \mathbb{R}_{+}$,

$$
\sup _{x \in(0, k), \theta}|q(x, \theta) / \sigma(x)|<+\infty .
$$


Proof. We use the Girsanov Theorem, as stated for example in Revuz-Yor [22] Chapter 8 Proposition 1.3 .

Let us consider the diffusion process $X^{k}$ on $[0, k]$, absorbed when it reaches 0 or $k$, at time $\tau_{k}:=T_{0}^{X} \wedge T_{k}^{X}$. The exponential martingale $\mathcal{E}\left(L^{k}\right)_{t}$, where $L_{t}^{k}=-\int_{0}^{t \wedge \tau_{k}} \frac{q\left(X_{s}, \theta_{s}\right)}{\sigma\left(X_{s}\right)} d B_{s}$, is uniformly integrable thanks to

(24) and Novikov's criterion. Define for any $x>0$ the probability $\mathbb{Q}_{x}$ with $\left.\frac{d \mathbb{Q}_{x}}{d \mathbb{P}_{x}}\right|_{\mathcal{F}_{t}}=\mathcal{E}(L)_{t}$. Then, the process $\omega=B-\langle B, L\rangle$ is a $\mathbb{Q}_{x}$-Brownian motion and, under $\mathbb{Q}_{x}, X$ is solution to the SDE $d X_{t}=$ $\sigma\left(X_{t}\right) d \omega_{t}+b\left(X_{t}\right) d t$. Hence $s$ restricted to $(0, k)$ is the scale function of $X^{k}$ under $\mathbb{Q}_{x}$. Since $s$ and $f$ are both bounded in a vicinity of $k$, we deduce from Theorem B.4 that

$$
\int_{0}^{\tau_{k}} f\left(X_{t}\right) d t<+\infty \text { a.s., under } \mathbb{Q}_{x}\left(\cdot \mid T_{k}^{X}<T_{0}^{X}\right) .
$$

Note also that, since we assumed that $T_{k}$ tends almost surely to infinity, we have up to a $\mathbb{P}_{x^{\prime}}$-negligible event,

$$
\left\{\int_{0}^{T_{0}} f\left(X_{t}\right) d t=+\infty\right\}=\bigcup_{k=0}^{+\infty}\left\{\int_{0}^{\tau_{k}} f\left(X_{t}\right) d t=+\infty\right\}
$$

and hence

$$
\mathbb{P}_{x}\left(\int_{0}^{T_{0}} f\left(X_{t}\right) d t=+\infty\right)=\lim _{k \rightarrow+\infty} \mathbb{P}_{x}\left(\int_{0}^{\tau_{k}} f\left(X_{t}\right) d t=+\infty\right)
$$

But, by definition of $\mathbb{Q}_{x}$ and by Theorem B.4, we have

$$
\begin{aligned}
\mathbb{P}_{x}\left(\int_{0}^{\tau_{k}} f\left(X_{t}\right) d t=+\infty\right) & =\mathbb{E}^{\mathbb{Q}_{x}}\left(\mathbb{1}_{\int_{0}^{\tau_{k}} f\left(X_{t}\right) d t=+\infty} \mathcal{E}\left(\int_{0}^{\tau_{k}} \frac{q\left(\omega_{s}, \theta_{s}\right)}{\sigma\left(\omega_{s}\right)} d \omega_{s}\right)\right) \\
& =\left\{\begin{array}{l}
0 \text { if } \int_{0+} s(y) f(y) m(d y)<+\infty \\
\mathbb{E}^{\mathbb{Q}_{x}}\left(\mathbb{1}_{T_{0}<T_{k}} \mathcal{E}\left(\int_{0}^{\tau_{k}} \frac{q\left(\omega_{s}, \theta_{s}\right)}{\sigma\left(\omega_{s}\right)} d \omega_{s}\right)\right) \text { otherwise }
\end{array}\right. \\
& =\left\{\begin{array}{l}
0 \text { if } \int_{0+} s(y) f(y) m(d y)<+\infty \\
\mathbb{P}_{x}\left(T_{0}<T_{k}\right) \text { otherwise. }
\end{array}\right.
\end{aligned}
$$

Letting $k$ tend to infinity concludes the proof.

\section{References}

[1] Anderson, W.J. Continuous-time Markov chains. Springer Series in Statistics: Probability and its applications. Springer-Verlag, New-York, 1991.

[2] Bansaye, V., Méléard, S. Stochastic models for structured populations, Mathematical Biosciences, Institute Lecture Series 1.4, Springer, 2015.

[3] Brown, J. H., Gillooly, J. F., Allen, A. P., Savage, V. M., and West, G. B. Toward a metabolic theory of ecology. Ecology, 85(7), (2004), 1771-1789.

[4] Byers, D. L., and D. M. Waller. Do plant populations purge their genetic load? Effects of population size and mating history on inbreeding depression. Annual Review of Ecology and Systematics 30.1 (1999): 479-513.

[5] Unifying evolutionary dynamics: from individual stochastic processes tomacroscopic models. N. Champagnat, R. Ferrière, S. Méléard. Theoretical Polulation Biology 69 (2006) 297-321.

[6] N. Champagnat. A microscopic interpretation for adaptive dynamics trait substitution sequence models. Stochastic Process. Appl., 116(8), (2006) 1127-1160. 
[7] N. Champagnat, D. Villemonais. General criteria for the study of quasi-stationarity. Arxiv e-prints, 1712.08092, 2018.

[8] C. Coron. Slow-fast stochastic diffusion dynamics and quasi-stationarity for diploid populations with varying size. J. Math. Biol. 72 (1-2), 171-202, 2016.

[9] C. Coron. Stochastic modeling and eco-evolution of a diploid population. PhD thesis under the supervision of Sylvie Méléard, Palaiseau, École Polytechnique, 2013. http://www.theses.fr/2013EPXX0052

[10] H.J. Engelbert, T. Senf. On Functionals of a Wiener Process with Drift and Exponential Local Martingales. Stochastic Processes and Related Topics, Series Mathematical Research, pp. 45 - 58, Akademie-Verlag, Berlin, Friedrich-Schiller-Univ., 1991.

[11] H.J. Engelbert, G. Tittel. Integral functionals of strong Markov continuous local martingale. In Stochastic Processes and Related Topics: Proceedings of the 12th Winter School Siegmundsburg, Germany, edited by R. Buckdahn, H.J. Engelbert and M. Yor. Taylor \& Francis, 2002.

[12] S.N. Ethier, T.G. Kurtz. Markov Processes. Characterization and convergence. Wiley series in statistics and probability. John Wiley \& Sons, 1986.

[13] C. Foucart, O. Hénard. Stable continuous stat branching processes with immigration and BetaFleming-Viot processes with immigration. Electron. J. Probab.18 (23), 1-21, 2013.

[14] Fournier, N. and Méléard, S. A microscopic probabilistic description of a locally regulated population and macroscopic approximations. Ann. Appl. Probab. 14(4), (2004), 1880-1919.

[15] Gillooly, J. F., Brown, J. H., West, G. B., Savage, V. M., and Charnov, E. L. Effects of size and temperature on metabolic rate. Science, 293(5538), (2001), 2248-2251.

[16] N. Ikeda, S. Watanabe. Stochastic differential equations and diffusion processes, 2nd edition, NorthHolland, 1989.

[17] O. Kallenberg. Foundations of modern probability, 2nd edition, Springer, 2001.

[18] I. Karatzas, S.E. Shreve. Brownian motion and stochastic calculus, 2nd edition, Springer, 1991.

[19] D. Khoshnevisan, P. Salminen and M. Yor. A note on a.s. finiteness of perpetual integral functionals of diffusions. Electron. Commun. Probab. 11, 108-117, 2006.

[20] M. Lynch, M. and W. Gabriel. Mutation load and the survival of small populations. Evolution 44:17251737, 1990.

[21] A. Mijatovic, M. Urusov. Convergence of integral functionals of one-dimensional diffusions. Electron. Commun. Probab. 17, 2012.

[22] D. Revuz, M. Yor. Continuous martingales and Brownian motion. Third edition, Springer, 1999.

[23] L.C.G. Rogers, D. Williams. Diffusions, Markov processes and martingales. Vol. 2, 2nd edition, Cambridge University Press, 2000.

[24] P. Salminen, M. Yor. Properties of perpetual integral functionals of Brownian motion with drift. Ann. Inst. Henri Poincaré Probab. Stat. 41(3), 335-347, 2005.

[25] Stroock, D.W., Varadhan, S.R.S.: Multidimensional diffusion processes. Springer.

[26] Svanbck R, Bolnick DI. Intraspecific competition drives increased resource use diversity within a natural population. Proceedings of the Royal Society B: Biological Sciences. 2007;274(1611):839844.

[27] West, G. B., Brown, J. H., and Enquist, B. J. The fourth dimension of life : fractal geometry and allometric scaling of organisms. Science, 284(5420), (1999), 1677-1679. 